\title{
Singularities and internal rotational dynamics of electron beams
}

\author{
D. Velasco-Martínez ${ }^{1}$, V. G. Ibarra-Sierra ${ }^{2}$, J. C. Sandoval-Santana ${ }^{2}$, J.L. Cardoso ${ }^{1}$, and A. Kunold ${ }^{1}$ \\ 1 Área de Física Teórica y Materia Condensada, \\ Universidad Autónoma Metropolitana Azcapotzalco, Av. San Pablo 180, \\ Col. Reynosa-Tamaulipas, Azcapotzalco, 02200 Cuidad de México, México \\ 2 Departamento de Física, Universidad Autónoma Metropolitana Iztapalapa, \\ Av. San Rafael Atlixco 186, Col. Vicentina, Iztapalapa, 09340 Cuidad de México, México
}

(Dated: October 19, 2018)

\begin{abstract}
We study the internal rotational dynamics of electronic beams in relation to the phase singularities of their wave functions. Given their complex singularity structure, Hermite-Gaussian beams and other superpositions of Laguerre-Gaussian modes are studied here. We show that by inspecting the lowest non-vanishing terms of the wave function near the singularity it is possible to infer the structure of the Bohmian streamlines. Conversely, starting from a map of the electron's Bohmian velocities, we demonstrate that it is possible to derive the form of the electron's wave function near the singularity. We outline a procedure that could yield an experimental method to determine the main parameters of the electron's wave function close to a singularity.
\end{abstract}

\section{INTRODUCTION}

The charged particle subject to a uniform and static magnetic field $[1-3]$ is at the heart of many physical theories. The quantum Hall [4] and related effects such as De Haas-Van Alphen effect [5], Shubnikov-De Haas oscillations [6] and the fractional quantum Hall effect [7] can not be understood without it. However, solid state systems are too complex to provide precise information of the internal dynamics of the Landau levels. Inspired by optical-vortex-beams, electron-vortex-beams have recently gained great attention [8-13] not only because they are expected to provide new capabilities to electron microscopes but also for allowing the observation of the electrons' isolated quantum states.

Whereas electron vortex beams may be understood as solutions of the Schrödinger equation, their optical counterparts are paraxial solutions of the Helmholtz equation [9-11]. In contrast to solid state systems, LaguerreGaussian (LG) beams allow for more precise and accurate observation of the internal dynamics of the Landau levels because they are generated in the rather controlled environment of a transmission electron microscope (TEM).

Among other techniques, electron vortex beams with helical wavefront carrying a large orbital angular momentum (as LG beams) are produced passing a standard electron beam through a holographic mask in TEMs [911, 14]. For example, LG beams are created by using a diffraction grating with a dislocation [11] that confers electrons a certain orbital angular momentum (OAM). In order to get actual Fock-Darwin states, the beam waist is chosen to match their diameter in a region where the axial magnetic field is uniform. When LG beams propagate along the direction of a uniform magnetic field, they behave as stable solutions of the Schrödinger equation. LG beams correspond in fact to the Fock-Darwin states that in turn are stationary solutions of the Shrödinger equation of a $2 \mathrm{D}$ charged particle in a magnetic field. Thereby most of their features remain constant and, as for their optical counterparts, the beam profile is main- tained as electrons propagate. Among these quantities, the OAM is preserved.

Bessel electron beams [15], Airy beams [16] and Hermite-Gaussian (HG) beams [17] have been studied and produced in the laboratory. HG modes, for example, may serve to probe the OAM of electron beams. All of these beams can be understood as linear combinations of LG states carrying different amounts of OAM.

Phase singularities play a crucial role in the formation of vortices in light. They have been widely studied since the pioneering work of Dirac [18], Aharonov, Bohm [19], Nye [20] and Berry [21]. Mathematically, optical singularities are regions in the domain of the electromagnetic field where the phase is indeterminate; as a consequence the field vanishes in these regions of space. Phase singularities are also present in electron beams and manifest themselves as vortices of density currents that turn around a zero of the wave function. Due to their cylindrical symmetry, LG beams can be made to have large OAM. Their wavefront spirals around a phase singularity located at the centre of the wave front [22, 23]. Singularities may be characterized by their topological charge, an integer number that counts the number of times the wave front makes a full revolution in one wavelength. In the case of LG beams and other eigenstates of the OAM the topological charge matches the OAM. Some linear combinations of LG modes are very interesting in this regard because, not carrying a distinctive angular momentum, they may present rich and complex singularity arrangements. Around each singularity, a density current vortex is formed whose properties fully depend on the mathematical form of the singularity.

Given the great amount of knowledge on optical vortices, they where the first candidates for OAM-based spectroscopic techniques. However, optical techniques have been proven to be very inefficient, owing to the weak optical multipolar transitions [24]. In contrast, it has been theoretical [25] and experimentally[13] demonstrated that the OAM of electron beams can be transferred to atomic electrons having an observable effect on 
the magnetic circular dichroism. It has also been suggested, that by creating different superpositions of LG beams [26], it is possible to tailor the internal electron currents. For instance, by superimposing various LG states it is possible to produce off-axis density current vortices [15]. This would allow addressing specific atoms in the sample by novel TEM techniques such as chiralspecific electron-vortex-beam spectroscopy [27].

In this paper we study the relation between the internal rotational dynamics of electron beams and their singularities. We study the features of the canonical and kinetic currents $[26,28]$ close to the phase singularities. We show that the properties of the electron density currents are completely determined by the singularities' structure and topological charge. To prove our results we have studied HG and related modes. The type of states that give rise to stable vortex excitations in Bose-Einstein condensates (BEC)[15] are also studied here given their interesting singularity structure.

Our results show that the mathematical structure of each singularity, completely determines the vortex structure in its vicinity. In particular, we demonstrate that it is possible to guess the shape of the Bohmian trajectories close to a singularity by inspecting the wave function's structure near it. Conversely, we show that it is possible to obtain the wave function's mathematical structure through the Bohmian streamlines around a vortex. This could lead to an experimental method to determine the electron's wave function close to a singularity.

This paper is organized as follows. In Sec. II we study Fock-Darwin states and introduce the rising and lowering operators that generate them. These operators are very practical for obtaining general expressions of the kinetic and canonical density currents in superpositions of LG modes. The mathematical form of $\mathrm{HG}$ and related modes as well as BEC states are introduced in Sec. III. Sec. IV presents the main results regarding the connection of the phase singularities' structure and the kinetic and canonical density currents. In Sec. VI we apply these results to the characterisation of the the singularities found in HG and BEC modes. We present the outline of a method to characterize the mathematical structure of singularities from the Bohmian streamlines in Sec. VII. In Sec. VIII we summarize the results and propose general conclusions.

\section{LG MODES. FOCK-DARWIN STATES}

The starting point is the Hamiltonian of an electron in a uniform magnetic field given by

$$
\hat{H}=\frac{1}{2 m}(\hat{\boldsymbol{p}}+e \hat{\boldsymbol{A}})^{2},
$$

where the vector potential in the symmetric gauge is given by

$$
\hat{\boldsymbol{A}}=-\frac{B}{2} \hat{y} \boldsymbol{i}+\frac{B}{2} \hat{x} \boldsymbol{j} .
$$

This particular choice makes the Hamiltonian (1) invariant under rotations. By expanding the momentum and position components, the Hamiltonian takes the form

$$
\hat{H}=\frac{1}{2 m}\left(\hat{p}_{x}^{2}+\hat{p}_{y}^{2}\right)+\frac{m \omega^{2}}{8}\left(\hat{x}^{2}+\hat{y}^{2}\right)+\frac{\omega}{2}\left(\hat{x} \hat{p}_{y}-\hat{y} \hat{p}_{x}\right),
$$

where $\omega=e B / m$ is the cyclotron frequency, the position and momentum operators follow the standard commutation relations $[\hat{x}, \hat{y}]=\left[\hat{p}_{x}, \hat{p}_{y}\right]=0$ and $\left[\hat{x}, \hat{p}_{x}\right]=\left[\hat{y}, \hat{p}_{y}\right]=$ $i \hbar$. In the equation above we immediately identify the $z$-component of the angular momentum $\hat{L}_{z}=\hbar l_{z}=$ $\hat{x} \hat{p}_{y}-\hat{y} \hat{p}_{x}$. A bit of algebra shows that it commutes with the Hamiltonian, i.e. $\left[\hat{H}, \hat{L}_{z}\right]=0$, and therefore must be a conserved quantity. This is a direct consequence of the adopted gauge.

To simplify the Hamiltonian we define the following rising and lowering operators

$$
\begin{aligned}
\hat{b} & =\frac{1}{2 \sqrt{2} l_{B}}\left[(\hat{x}+i \hat{y})+i \frac{2}{m \omega}\left(\hat{p}_{x}+i \hat{p}_{y}\right)\right], \\
\hat{b}^{\dagger} & =\frac{1}{2 \sqrt{2} l_{B}}\left[(\hat{x}-i \hat{y})-i \frac{2}{m \omega}\left(\hat{p}_{x}-i \hat{p}_{y}\right)\right], \\
\hat{c} & =\frac{1}{2 \sqrt{2} l_{B}}\left[(\hat{x}-i \hat{y})+i \frac{2}{m \omega}\left(\hat{p}_{x}-i \hat{p}_{y}\right)\right], \\
\hat{c}^{\dagger} & =\frac{1}{2 \sqrt{2} l_{B}}\left[(\hat{x}+i \hat{y})-i \frac{2}{m \omega}\left(\hat{p}_{x}+i \hat{p}_{y}\right)\right],
\end{aligned}
$$

where $l_{B}=\sqrt{\hbar / m \omega}$ is the magnetic length. It can readily be verified that they follow the usual commutation rules $\left[\hat{b}, \hat{b}^{\dagger}\right]=\left[\hat{c}, \hat{c}^{\dagger}\right]=1$ and $[\hat{b}, \hat{c}]=\left[\hat{b}^{\dagger}, \hat{c}^{\dagger}\right]=0$. From the definitions given above, the Hamiltonian can be expressed in the compact form of a quantum oscillator

$$
\hat{H}=\hbar \omega\left(\hat{c}^{\dagger} \hat{c}+\frac{1}{2}\right)
$$

The number operators $\hat{b}^{\dagger} \hat{b}$ and $\hat{c}^{\dagger} \hat{c}$ commute and therefore have simultaneous eigenstates. We therefore use their eigenvalues to label the kets $|l, n\rangle$, where $\hat{c}^{\dagger} \hat{c}|l, n\rangle=$ $l|l, n\rangle$ and $\hat{b}^{\dagger} \hat{b}|l, n\rangle=n|l, n\rangle$. Additionally, from the properties of quantum oscillators, we know that $l, n=$ $0,1,2 \ldots \infty$. Given that $\hat{b}$ and $\hat{c}$ follow the standard commutation rules of quantum oscillators, we can take advantage of their properties as rising and lowering operators and express any normalized state of the charged particle as

$$
|l, n\rangle=\frac{\left(c^{\dagger}\right)^{l}\left(\hat{b}^{\dagger}\right)^{n}}{\sqrt{l ! n !}}|0,0\rangle
$$

where $|0,0\rangle$ is the ground-state. These are the FockDarwin states. They are degenerate energy eigenstates with energy eigenvalues given by $E_{l}=\hbar \omega(l+1 / 2)$. Here it is clear that $l$ tags the Landau levels and $n$ parametrizes 
the degeneracy. The angular momentum may be conveniently expressed in terms of the rising and lowering operators (4)-(7) as

$$
\hat{l}_{z}=\hat{c}^{\dagger} \hat{c}-\hat{b}^{\dagger} \hat{b}
$$

Since $\hat{l}_{z}$ depends on the number operators, the state $|l, n\rangle$ is also an eigenstate of the angular momentum, i.e. $\hat{l}_{z}|l, n\rangle=m|l, n\rangle=(l-n)|l, n\rangle$ where $m$ is the angular momentum eigenvalue. As $n$ and $l$ are positive integers, the angular momentum $m$ might be negative, in contrast to the classical case, where the angular momentum is always positive. One of the most striking implications of this fact is the existence of diamagnetic states with negative OAM.

The very well known position-space wave function of Fock-Darwin states is given by

$$
\begin{aligned}
& \psi_{l, n}^{L G}\left(z, z^{*}\right)=\frac{1}{\sqrt{2 \pi} l_{B}} \exp \left(-\frac{z z^{*}}{4 l_{B}^{2}}\right)
\end{aligned}
$$

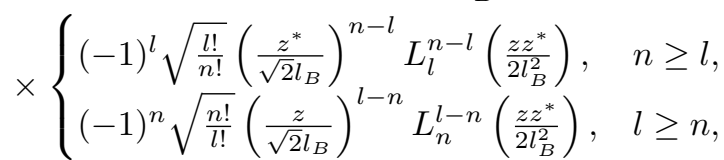

where $z=x+i y$. The corresponding probability density is given by

$$
\begin{aligned}
\rho_{l, n}^{L G}(r)= & \frac{1}{2 \pi l_{B}^{2}} \frac{\min (l, n) !}{\max (l, n) !}\left(\frac{r^{2}}{2 l_{B}^{2}}\right)^{|l-n|} \\
& \times \exp \left(-\frac{r^{2}}{2 l_{B}^{2}}\right)\left[L_{\min (l, n)}^{|l-n|}\left(\frac{r^{2}}{2 l_{B}^{2}}\right)\right]^{2},
\end{aligned}
$$

where $z=r \exp (i \theta)$ and $r^{2}=x^{2}+y^{2}=|z|^{2}$. These are precisely the LG modes.

\section{SUPERPOSITION OF LG MODES.}

Any beam can in principle be expressed as a linear superposition of LG mode as

$$
\psi(x, y)=\sum_{l, n} A_{l, n} \psi_{l, n}^{L G}(x, y),
$$

where

$$
A_{l, n}=\langle l, n \mid \psi\rangle=\int d x d y \psi_{l, n}^{L G *}(x, y) \psi(x, y) .
$$

A particular case are HG beams. HG electron beams have been generated by means of mode converters [17] that add or subtract units of the topological charge to an incident beam. Therefore a LG mode having nonvanishing angular momentum and topological charge can be turned into a HG mode with vanishing topological charge. An immediate application of mode converters would then be mode discrimination. Adding one charge unit to a given negatively charged vortex would allow to distinguish it from its positively charged twin by comparing the otherwise identical spatial intensity distributions [17].

HG beams can be thought of as stationary solutions of a two-dimensional isotropic oscillator. Their wave function is given by

$$
\begin{array}{r}
\psi_{j, k}^{H G}(x, y)=\frac{1}{\sqrt{2 \pi 2^{j} 2^{k} j ! k ! l_{B}}} \exp \left(-\frac{x^{2}+y^{2}}{4 l_{B}^{2}}\right) \\
\times H_{j}\left(\frac{x}{\sqrt{2} l_{B}}\right) H_{k}\left(\frac{y}{\sqrt{2} l_{B}}\right) .
\end{array}
$$

Using the relation between Hermite and Laguerre 2D polynomials[29]

$$
\begin{aligned}
& H_{j}(x) H_{k}(y)=i^{k} \sum_{q=0}^{j+k} 2^{q} P_{q}^{(j-q, k-q)}(0) \\
& \times\left\{\begin{array}{r}
(-1)^{q} q !(x-i y)^{j+k-2 q} \\
\times L_{q}^{j+k-2 q}\left(x^{2}+y^{2}\right), \quad 2 q \leq j+k, \\
(-1)^{j+k-q}(j+k-q) !(x+i y)^{2 q-j-k} \\
\times L_{j+k-q}^{2 q-j-k}\left(x^{2}+y^{2}\right), \quad 2 q \geq j+k,
\end{array}\right.
\end{aligned}
$$

one can identify the $A_{l, n}$ coefficients for HG beams. Their wave functions adopt the form

$$
\psi_{j, k}^{H G}(x, y)=\sum_{q=0}^{j+k} A_{q}^{j, k} \psi_{q, j+k-q}^{L G}(x, y),
$$

where

$$
A_{q}^{j, k}=2^{q} i^{k} \sqrt{\frac{q !(j+k-q) !}{2^{j} 2^{k} j ! k !}} P_{q}^{(j-q, k-q)}(0),
$$

and $P_{q}^{j-q, k-q}(0)$ are the Jacobi polynomials. From Eq. (17) we can readily calculate the time-dependent HG states

$$
\begin{aligned}
& \psi_{j, k}^{H G}(x, y, t) \\
& =\sum_{q=0}^{j+k} A_{q}^{j, k} \exp \left[-i \omega\left(q+\frac{1}{2}\right) t\right] \psi_{q, j+k-q}^{L G}(x, y) .
\end{aligned}
$$

In general, HG beams have the structure of balanced states [30] i.e. they are formed of linear combinations of LG beams where states having opposite angular momentum participate with the same weight. Thereby HG beams have vanishing overall angular momentum. However, it is possible to separate HG beams in a negative, vanishing and positive part as

$$
\psi_{i, j}^{H G}=\psi_{i, j}^{-H G}+\psi_{i, j}^{0 H G}+\psi_{i, j}^{+H G} .
$$

These are correspondingly formed of LG states having negative, vanishing and positive angular momenta. Nevertheless, they are not necessarily angular momentum 


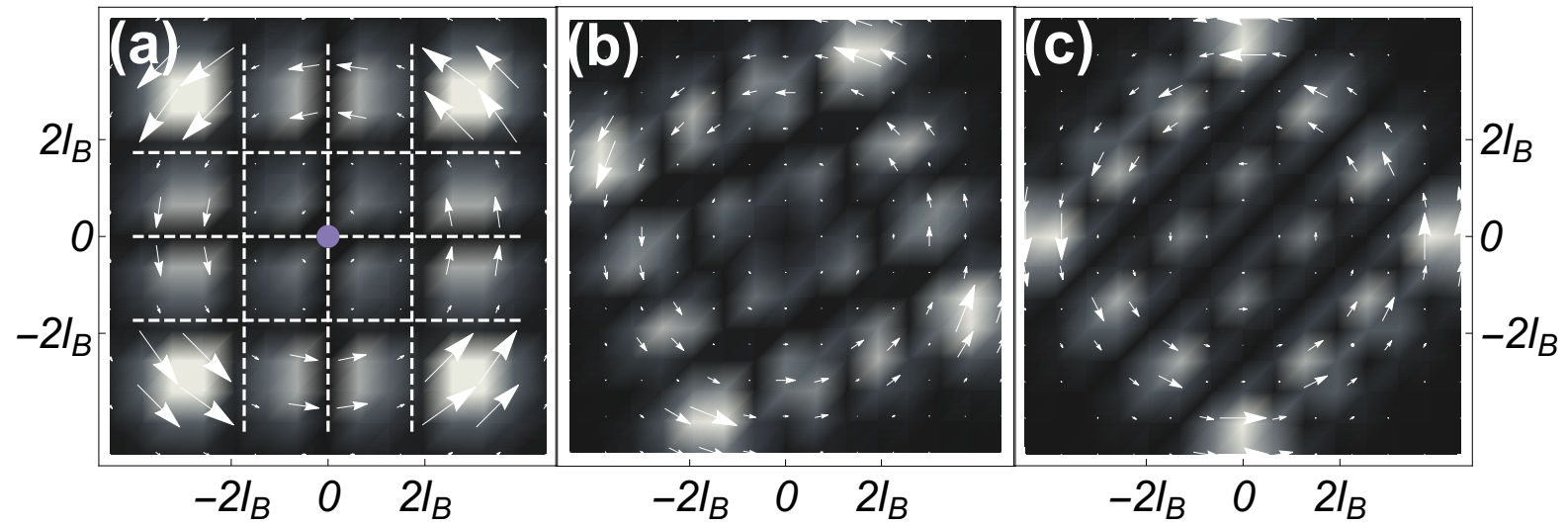

FIG. 1. Rotational dynamics of $\psi_{3,3}^{H G}$. A vector field of the kinetic density current is superimposed to the density plot to the probability density $\rho_{3,3}^{H G}$. In (a) we observe the probability density $\rho_{3,3}^{H G}$ at $t=0$. The dashed lines and the (purple) point indicate the position of the singularities $S_{3,3}^{H G}$. The Figs. (b) and (c) show the evolution of the probability density at $t=\pi / 4 \omega$ and $t=\pi / 2 \omega$ respectively.
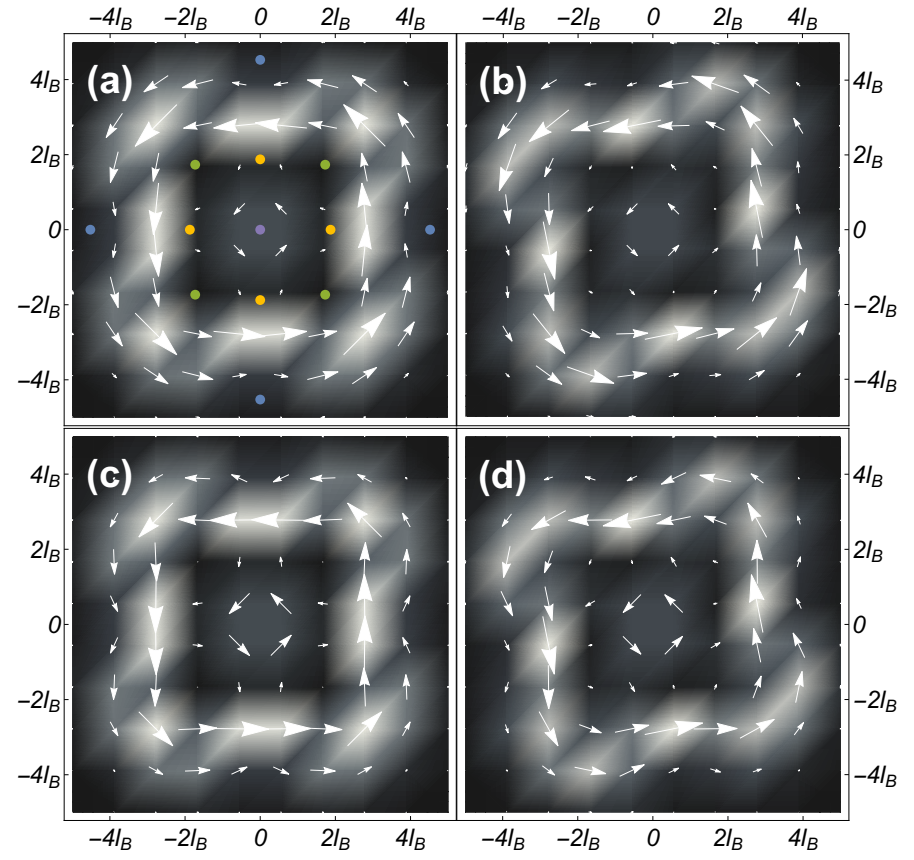

FIG. 2. Rotational dynamics of $\psi_{3,3}^{+H G}$. Along the first row, in (a) and (b) we observe the time evolution of $\rho_{3,3}^{+H G}$ and the vector field $\boldsymbol{J}^{K}$ for $t=0$ and $t=\pi / 8 \omega$ respectively. In the second row, (c) and (d) show the time evolution of $\rho_{3,3}^{+H G}$ and the vector field $\boldsymbol{J}^{C}$ for $t=0$ and $t=\pi / 8 \omega$ respectively. The positions of the different kinds of singularities are indicated with (colored) dots in (a).

eigenstates. In pure HG beams phase singularities are arranged as lines with vanishing topological charge. Conversely, the negative and positive parts of the HG states possess phase singularities arranged as isolated points with different topological charges. We are particularly

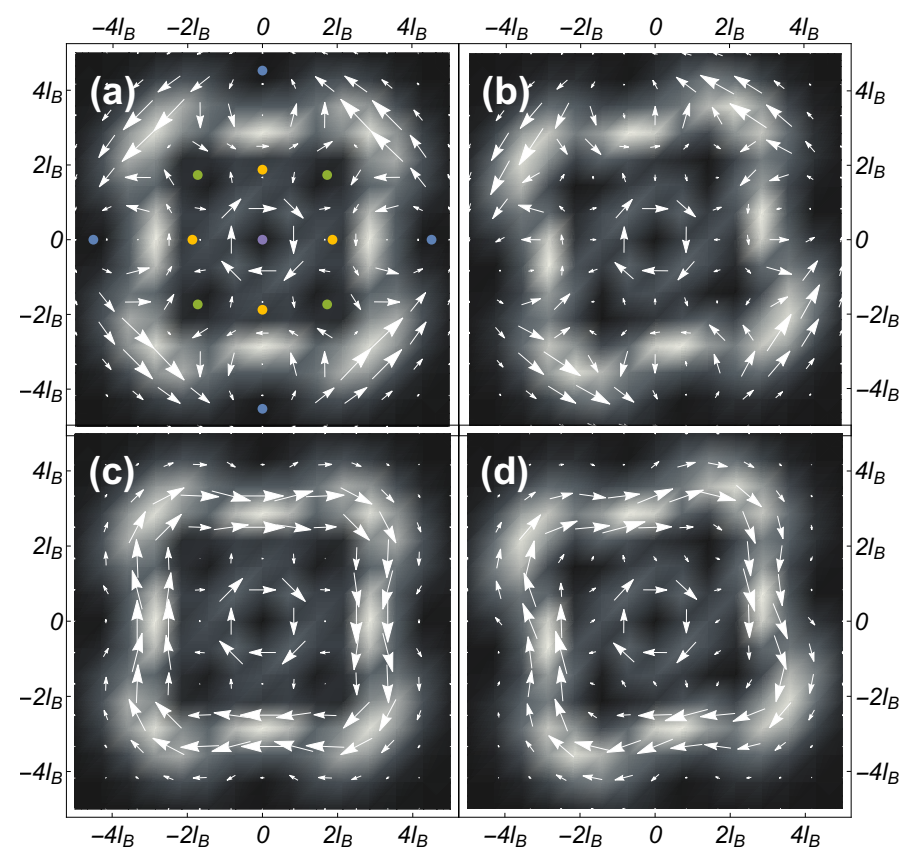

FIG. 3. Internal rotational dynamics of $\psi_{3,3}^{-H G}$. Along the first row, in (a) and (b) we observe the time evolution of $\rho_{3,3}^{-H G}$ and the vector field $\boldsymbol{J}^{K}$ for $t=0$ and $t=\pi / 8 \omega$ respectively. In the second row, (c) and (d) show the time evolution of $\rho_{3,3}^{-H G}$ and the vector field $\boldsymbol{J}^{C}$ for $t=0$ and $t=\pi / 8 \omega$ respectively. The positions of the different kinds of singularities are indicated with (colored) dots in (a).

interested in these states because of their rich and complex singularity structure. 


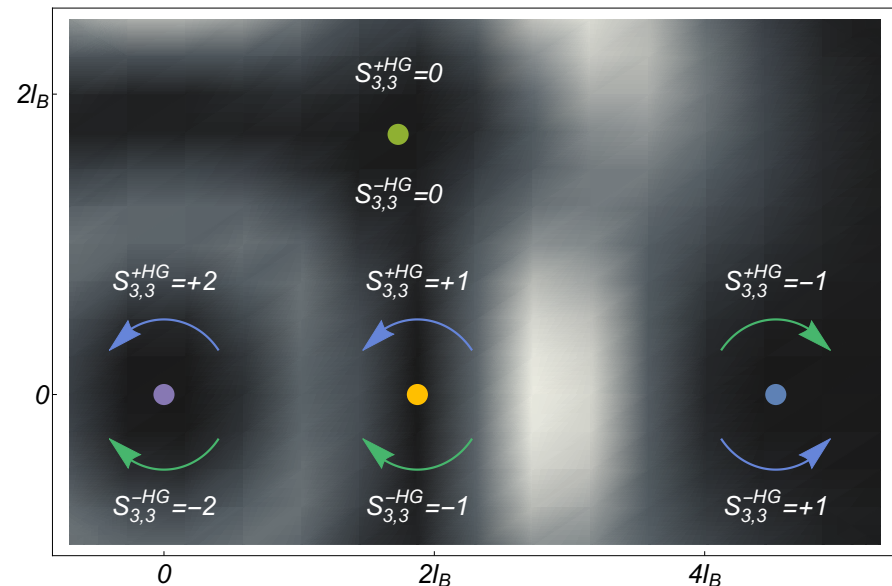

FIG. 4. Position and topological charges of the $\psi_{3,3}^{+H G}$ and $\psi_{3,3}^{-H G}$ singularities. The position and spinning directions of the main types of singularities are superimposed to the density plot to the probability density $\rho_{3,3}^{ \pm H G}$. These singularities are also shown in Figs. 2 and 1.

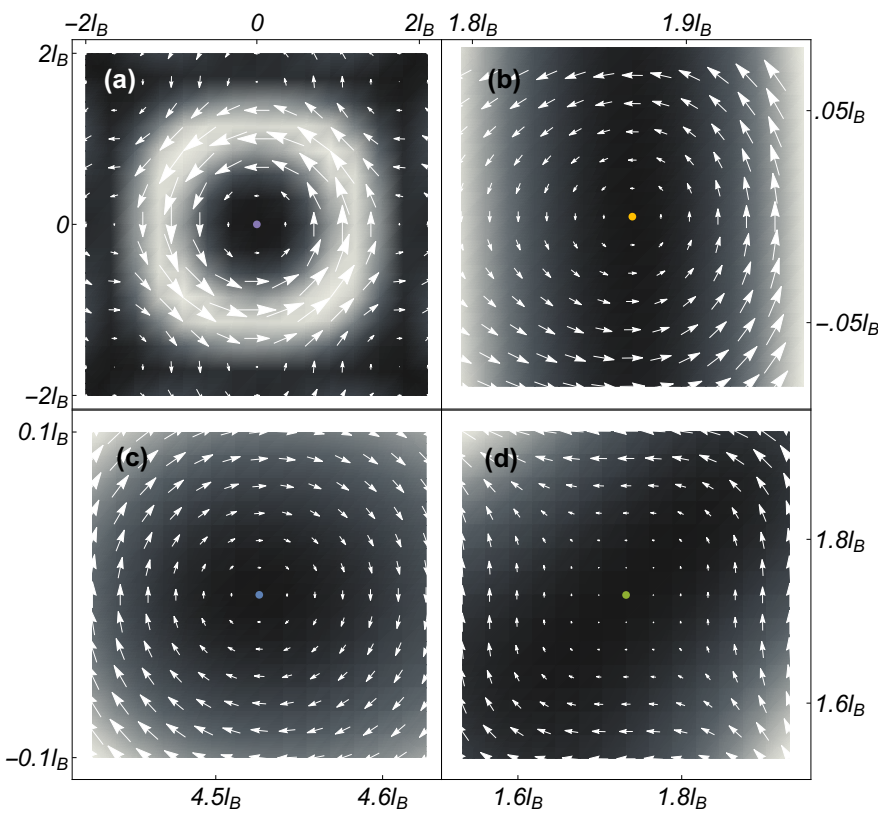

FIG. 5. Singularities of $\psi_{3,3}^{+H G}$. The positions of the four main types of $\psi_{3,3}^{+H G}$ singularities are indicated with (colored) dots. The density plot of the density probability $\rho_{3,3}^{+H G}$ along with the corresponding vector field $\boldsymbol{J}^{C}$ are shown. (a), (b), (c) and (d) show the vorticity of the $S_{3,3}^{+H G}=+2, S_{3,3}^{+H G}=+1$, $S_{3,3}^{+H G}=-1$ and $S_{3,3}^{+H G}=0$ topological charges, respectively.

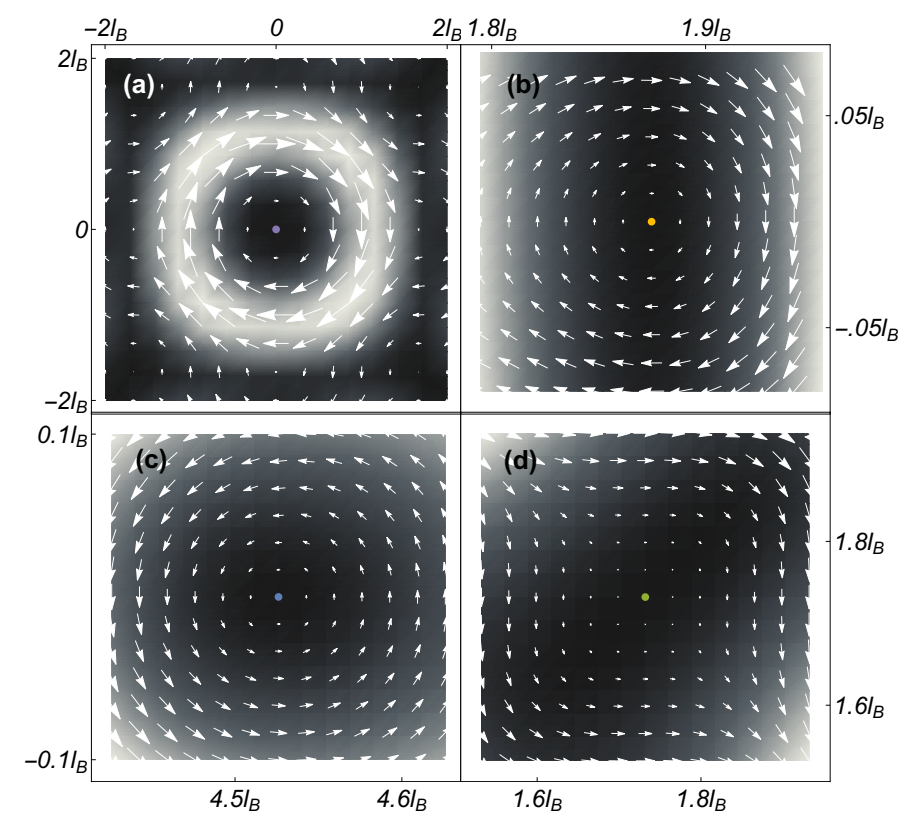

FIG. 6. Singularities of $\psi_{3,3}^{-H G}$. The positions of the four main types of $\psi_{3,3}^{-H G}$ singularities are indicated with (colored) dots. The density plot of the density probability $\rho_{3,3}^{-H G}$ along with the corresponding vector field $\boldsymbol{J}^{C}$ are also shown. (a), (b), (c) and (d) show the vorticity of the $S_{3,3}^{-H G}=-2, S_{3,3}^{-H G}=-1$, $S_{3,3}^{-H G}=+1$ and $S_{3,3}^{-H G}=0$ topological charges, respectively.
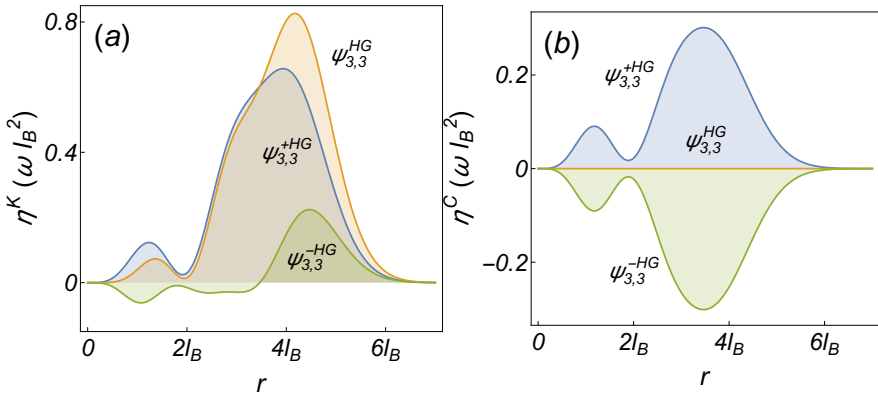

FIG. 7. $\eta^{K}$ and $\eta^{C}$ for $\psi_{3,3}^{H G}$ (orange), $\psi_{3,3}^{+H G}$ (blue) and $\psi_{3,3}^{-H G}$ (green) as functions of $r=\sqrt{x^{2}+y^{2}}$. In the negative beam $\psi_{3,3}^{-H G} \eta^{K}$ changes sign and $\eta^{C}$ is symmetric with respect to the positive beam $\psi_{3,3}^{+H G}$.

The vanishing part of an HG beam is given by

$$
\begin{gathered}
\psi_{j, k}^{0 H G}(x, y, t)=A_{(j+k) / 2}^{j, k} \exp \left(-i \frac{j+k}{2} \omega t\right) \\
\times \begin{cases}\psi_{(j+k) / 2,(j+k) / 2}^{L G}(x, y), & i \text { and } j \text { even numbers }, \\
0, & i \text { or } j \text { odd numbers } .\end{cases}
\end{gathered}
$$




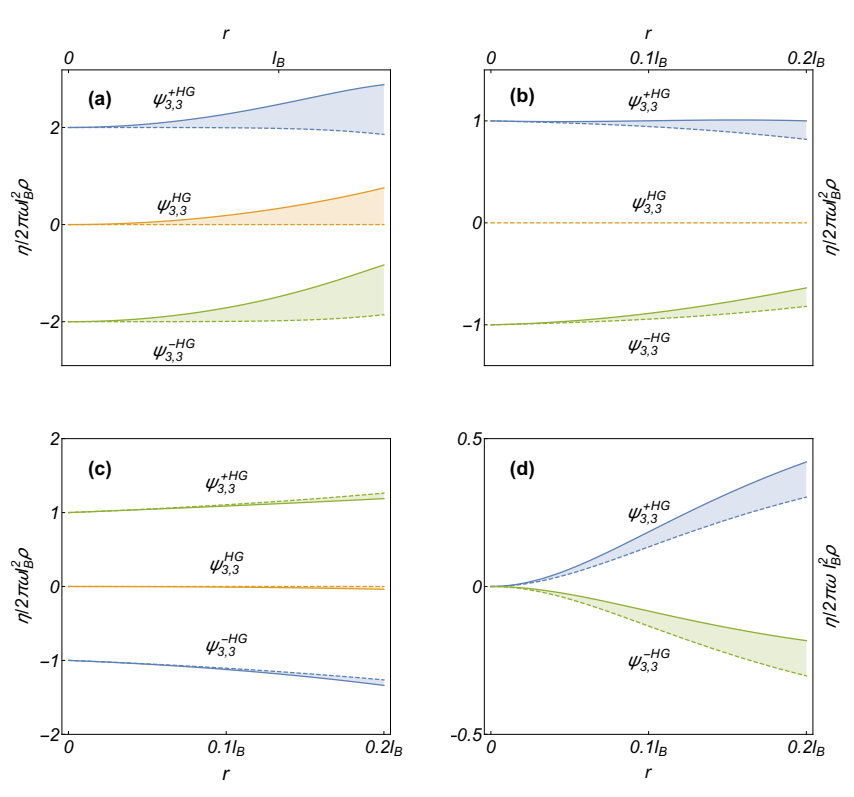

FIG. 8. Topological charges of the $\psi_{3,3}^{-H G}$ and $\psi_{3,3}^{+H G}$ singularities. $\eta^{K} / 2 \pi \omega l_{B}^{2} \rho$ (solid lines) and $\eta^{C} / 2 \pi \omega l_{B}^{2} \rho$ (dashed lines) are ploted as functions of $r$, the distance to the singularity. (a), (b), (c) and (d) show $\eta^{C} / 2 \pi l_{B}^{2} \rho$ and $\eta^{K} / 2 \pi l_{B}^{2} \rho$ corresponding to the $S_{3,3}^{ \pm H G}= \pm 2, S_{3,3}^{ \pm H G}= \pm 1, S_{3,3}^{\mp H G}=\mp 1$ and $S_{3,3}^{ \pm H G}=0$ singularities respectively. The singularities (a), (c) and (d) are approached along the angle $\theta=\pi / 3$, and the one in (b) is approached along $\theta=0$.

The positive and negative parts can be expressed as

$$
\psi_{j, k}^{ \pm H G}(x, y, t)=\sum_{q=q_{m i n}^{ \pm}}^{q_{m a x}^{ \pm}} A_{q}^{j, k} \mathrm{e}^{-i q \omega t} \psi_{q, j+k-q}^{L G}(x, y),
$$

where

$$
\begin{aligned}
& q_{\text {min }}^{-}=0, \\
& q_{\text {max }}^{-}=\operatorname{int}\left(\frac{j+k}{2}\right)-p, \\
& q_{\text {min }}^{+}=\operatorname{int}\left(\frac{j+k}{2}\right)+p, \\
& q_{\text {max }}^{+}=j+k,
\end{aligned}
$$

with $p=1$ if $i$ and $j$ are even, and $p=0$ if $i$ or $j$ are odd. Pure HG beams are generated for $q_{\text {min }}=0$ and $q_{\max }=j+k$. by

Other superpositions of LG modes, as the ones given

$$
\begin{aligned}
\psi_{n}^{+B E C} & =\frac{1}{\sqrt{5}} \psi_{0,0}^{L G}+\frac{2}{\sqrt{5}} \psi_{n, 0}^{L G}, \\
\psi_{n}^{-B E C} & =\frac{1}{\sqrt{5}} \psi_{0,0}^{L G}+\frac{2}{\sqrt{5}} \psi_{0, n}^{L G},
\end{aligned}
$$

yield interesting singularity arrangements. It has been proved that their optical analogues have $n$ vortices located at equally spaced points around the center. Their

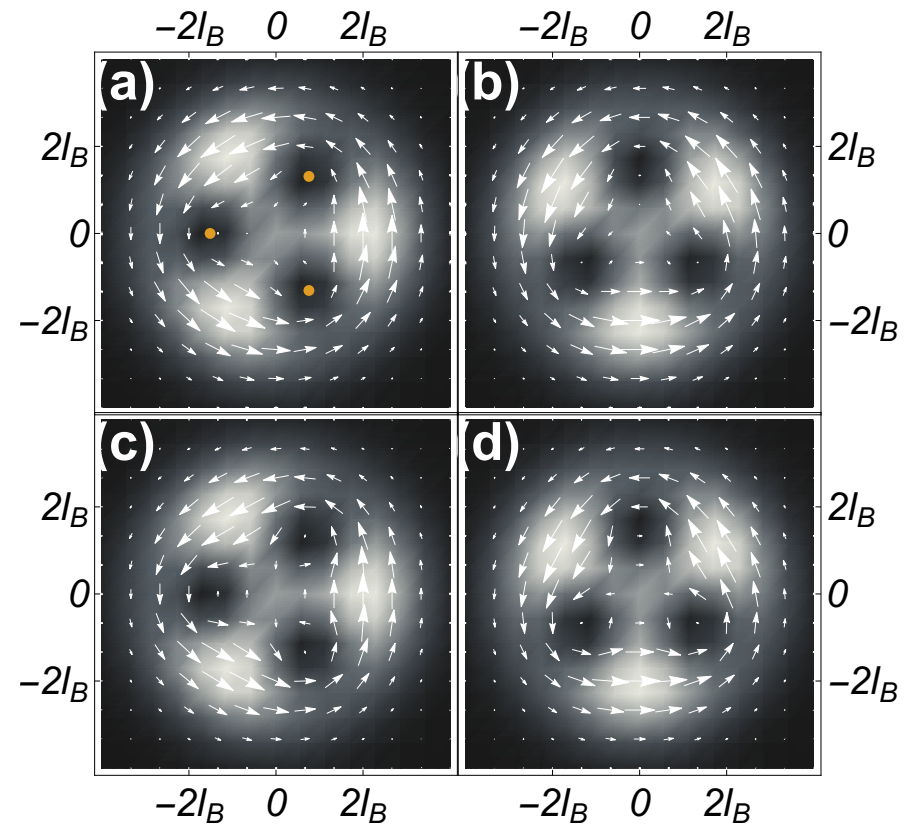

FIG. 9. Rotational dynamics of $\psi_{3}^{+B E C}$. Along the first row, in (a) and (b) we observe the time evolution of $\rho_{3}^{+B E C}$ and the vector field $\boldsymbol{J}^{K}$ for $t=0$ and $t=\pi / 6 \omega$ respectively. In the second row, (c) and (d) show the time evolution of $\rho_{3}^{+B E C}$ and the vector field $\boldsymbol{J}^{C}$ for $t=0$ and $t=\pi / 6 \omega$ respectively. The positions of the three singularities are indicated with (orange) dots in (a).

vortex structure has been studied in optical beams [31] and plays an important role in Bose-Einstein condensates stirred with a laser beam $[30,32]$.

\section{TOPOLOGICAL CHARGE OF ELECTRON BEAMS}

Any point in the wave function's domain where the amplitude vanishes presents a singularity where the phase is undefined. These are also called vortex singularities because the wave function's phase circulates around these points. The singularity of a state $\psi=|\psi| \mathrm{e}^{i \phi}$ may be characterized by its topological charge

$$
S=\frac{1}{2 \pi} \oint_{\mathcal{C}} \nabla \phi \cdot d \boldsymbol{l}=\frac{1}{2 \pi} \int_{0}^{2 \pi} \frac{d \phi}{d \theta} d \theta
$$

where $\mathcal{C}$ is a closed path around a point where $\psi=$ $|\psi| \exp (i \phi)$ vanishes.

In the particular case of LG beams the calculation of the topological charge is straightforward. These states only possess one singularity located in the symmetry axis of the beam $(z=0)$. It can be easily verified that the Fock-Darwin states in Eq. (11) have an $(n-l)$-fold zero in $z=0$. In this point the LG modes vanish if $l \neq n$ and consequently the phase is undefined. By setting $z=$ $r \exp (i \theta)$ in Eq. (11) we notice that the wave function's 


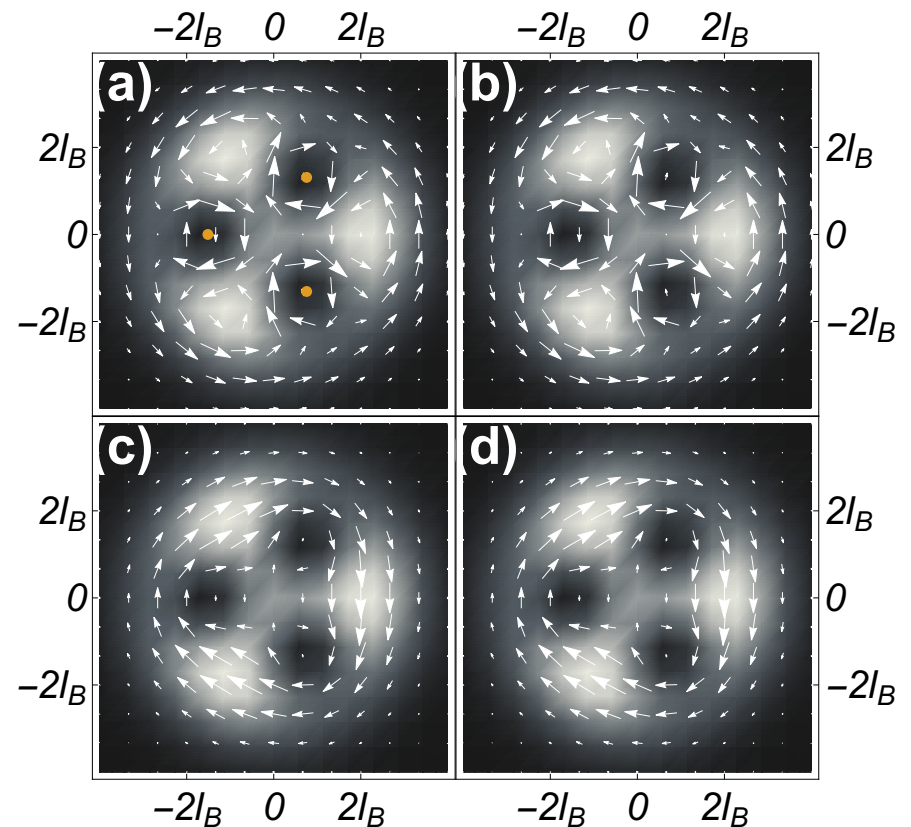

FIG. 10. Rotational dynamics of $\psi_{3}^{-B E C}$. Along the first row, in (a) and (b) we observe the time evolution of $\rho_{3}^{-B E C}$ and the vector field $\boldsymbol{J}^{K}$ for $t=0$ and $t=\pi / 6 \omega$ respectively. The singularities clearly revolve around the center in the counterclockwise direction. In the second row, (c) and (d) show the time evolution of $\rho_{3}^{-B E C}$ and the vector field $\boldsymbol{J}^{C}$ for $t=0$ and $t=\pi / 6 \omega$ respectively. The positions of the three singularities are indicated with (orange) dots in (a).

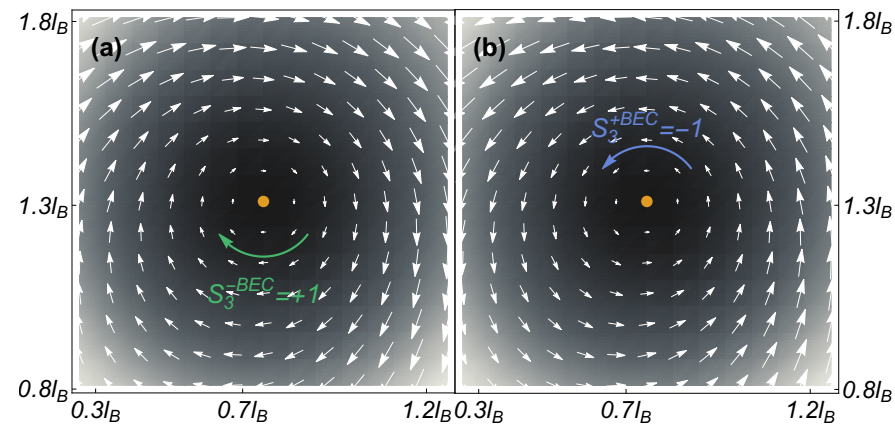

FIG. 11. Topological charges and positions of the (a) $\psi_{3}^{+B E C}$ and (b) $\psi_{3}^{-B E C}$ singularities. The position and the kinetic density currents' spinning direction of the singularities are superimposed to the density plot to the probability density $\rho_{3}^{ \pm B E C}$. The vector plot indicates the vector field of the kinetic density current $\boldsymbol{J}^{K}$. These singularities are also shown in Figs. 9 and 10.

phase is $\phi=(l-n) \theta$ and, from Eq. (29), the topological charge is given by

$$
S_{l, n}^{L G}=(l-n)
$$

Thus, the topological charge of the singularity in LG states is identical to the angular momentum. The struc-
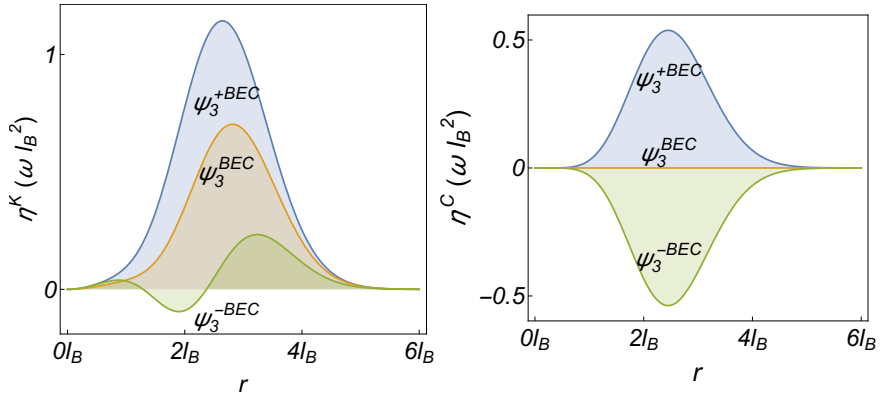

FIG. 12. $\eta^{K}$ and $\eta^{C}$ for $\psi_{3}^{B E C}$ (orange), $\psi_{3}^{+B E C}$ (blue) and $\psi_{3}^{-B E C}$ (green) as functions of $r=\sqrt{x^{2}+y^{2}}$. In the negative beam $\psi_{3}^{-B E C} \eta^{K}$ changes sign and $\eta^{C}$ is symmetric with respect to the positive beam $\psi_{3}^{+B E C}$.

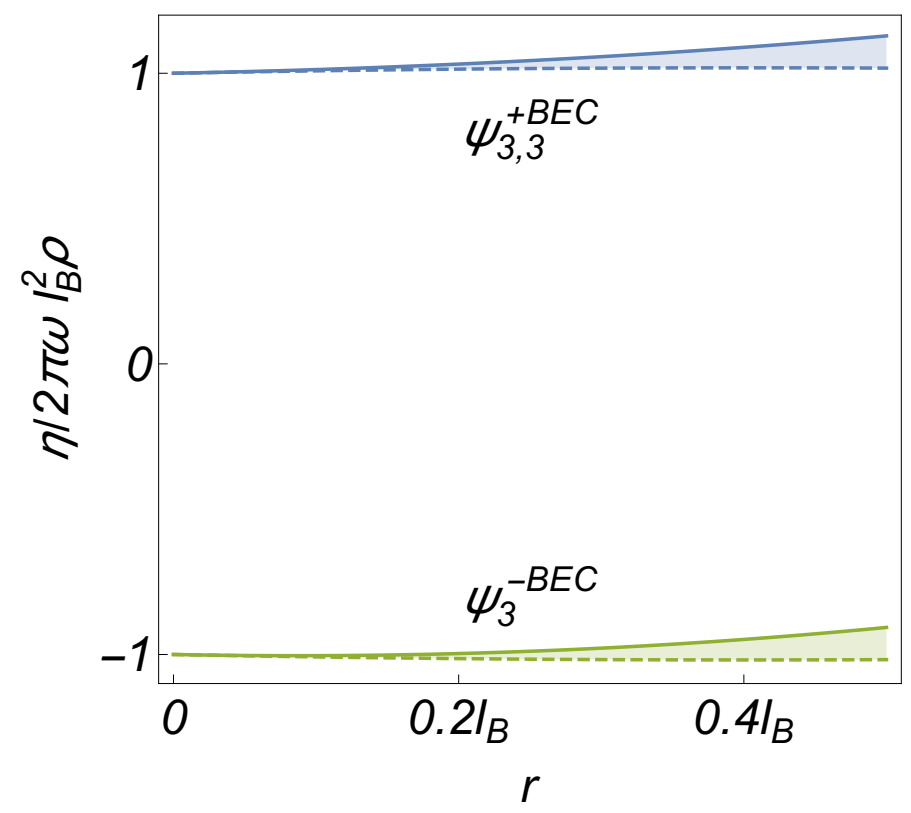

FIG. 13. Topological charges of the $\psi_{3}^{-B E C}$ and $\psi_{3}^{+B E C}$ singularities. $\eta^{K} / 2 \pi \omega l_{B}^{2} \rho$ (solid lines) and $\eta^{C} / 2 \pi \omega l_{B}^{2} \rho$ (dashed lines) are plotted as functions of $r$, the distance to the singularity. The plot is shown for the $S_{3}^{ \pm B E C}= \pm 1$ singularity located in $z=(2 \sqrt{3})^{1 / 3} \exp (i \pi / 3)$. The singularity is approached along the angle $\theta=0$.

ture of the LG modes in the vicinity of the singularity are listed in rows 1-2 of Table I.

In beams whose wave functions are not eigenstates of the angular momentum, singularities may have a more involved structure. An arbitrary state $\psi$ may have a number of phase singularities $z_{1}=x_{1}+i y_{1}, z_{2}=x_{2}+i y_{2}$, $\ldots, z_{n}=x_{n}+i y_{n}$. Expanding the wave function around one of them, say $z_{j}$, up to the lowest non-trivial power we obtain

$$
\psi=a\left[\gamma X^{\prime}+i \beta Y^{\prime}\right]^{s} .
$$

The coordinates $X=x-x_{j}$ and $Y=y-y_{j}$ belong to a frame oriented along the singularity's symmetry axes 
and whose origin is located in the singularity. If the singularity's symmetry axes form an $\nu$ angle with respect to the standard frame, then $X^{\prime}=\cos \nu X-\sin \nu Y$, $Y^{\prime}=\cos \nu Y+\sin \nu X$ where $X^{\prime}$ and $Y^{\prime}$ are in the standard reference frame. The $\gamma$ and $\beta$ parameters are real and $a=|a| \exp (i \alpha)$ is a complex number. The wave function will have elliptical symmetry and in general it will not be aligned with the standard reference frame. Although the wave function may have other forms in the vicinity of a singularity, (31) is general enough to allow the analysis of the wave functions presented here.

In order to perform a closed integral (29) around the singularity and since in general $\gamma \neq \beta$ it is convenient to set

$$
\bar{z}-\bar{z}_{j}=|\gamma|\left(x-x_{j}\right)+i|\beta|\left(y-y_{j}\right)=r \exp (i \varphi) .
$$

Performing the closed integral around a circle in the $\bar{z}$ variable $(0 \leq \varphi \leq 2 \pi)$ is equivalent to integrating the original $z$ variables around an ellipse $(0 \leq \theta \leq 2 \pi)$ centered in $z_{j}$ and whose semi-major and semi-minor axes are $\gamma$ and $\beta$.

Three different cases must be considered: 1) $\gamma \beta>0$, 2) $\gamma \beta<0$ and 3) $\gamma \beta=0$. If $\gamma \beta>0$ then the wave function (31) can be approximated by $\psi= \pm|a| \bar{z}^{s}=$ $\pm|a| r^{s} \exp (i \alpha+s \varphi)$. Therefore the wave function's phase in $\bar{z}=\bar{z}_{j}$ is $\phi=(1 \mp 1) \pi / 2+\alpha+s \varphi$. Inserting the phase in the integral (29), the topological charge yields

$$
S=\frac{1}{2 \pi} \int d \phi=\frac{1}{2 \pi} s \int_{0}^{2 \pi} d \varphi=s, \quad \gamma \beta>0 .
$$

Similarly, if $\gamma \beta<0$ the singularity is located in the isolated point $\bar{z}^{*}=\bar{z}_{j}^{*}$. Thereby the first non-vanishing term of the wave function expansion is given by $\psi=$ $\pm|a|\left(\bar{z}^{*}\right)^{s}= \pm|a| r^{s} \exp (i \alpha-s \varphi)$ and the topological charge yields

$$
S=\frac{1}{2 \pi} \int d \phi=-\frac{1}{2 \pi} s \int_{0}^{2 \pi} d \varphi=-s, \quad \gamma \beta<0 .
$$

We have quite a different situation when $\gamma \beta=0$. In this case, the phase of the wave function close to the singularity is constant. If $\gamma=0$ then $\phi=\pi / 2$ and if $\beta=0$ then $\phi=0$. In either case the phase is constant and therefore

$$
S=\frac{1}{2 \pi} \int d \phi=0, \quad \gamma \beta=0 .
$$

\section{KINETIC AND CANONICAL CURRENTS}

In most quantum mechanical systems the canonical momentum $\hat{\boldsymbol{p}}$ is proportional to the velocity operator $\hat{\boldsymbol{v}}$. However, when the effect of the magnetic field is introduced, this is no longer true due to extra terms arising from the vector potential $\boldsymbol{A}$. These terms strongly depend on the gauge choice. One can then distinguish two different kinds of momenta: the canonical momentum $\hat{\boldsymbol{p}}$ and the kinetic momentum $m \hat{\boldsymbol{v}}[26,28]$. One can accordingly compute two types of density current: the canonical density currents

$$
\begin{array}{r}
\boldsymbol{J}^{C}=\operatorname{Re}\left[\psi^{*}(x, y, t)\left(-i \frac{\hbar}{m} \nabla\right) \psi(x, y, t)\right] \\
=\operatorname{Re}\left[\psi^{*}(x, y, t)\left\langle x, y\left|\frac{\hat{\boldsymbol{p}}}{m}\right| \psi\right\rangle\right]
\end{array}
$$

and the kinetic density current

$$
\begin{array}{r}
\boldsymbol{J}^{K}=\operatorname{Re}\left[\psi^{*}(x, y, t)\right. \\
\left.=\operatorname{Re}\left[\frac{\hbar}{m} \nabla+\frac{e}{m} \boldsymbol{A}\right) \psi(x, y, t)\right] \\
=\operatorname{Re}\left[\psi^{*}(x, y, t)\langle x, y|\hat{\boldsymbol{v}}| \psi\rangle\right]
\end{array}
$$

where $\hat{\boldsymbol{v}}=(\hat{\boldsymbol{p}}+e \hat{\boldsymbol{A}}) / m$ is the velocity operator. These currents, specially the kinetic one, are of great experimental significance. The kinetic density current is associated to the Bohmian velocity by $\boldsymbol{v}^{K}=\boldsymbol{J}^{K} / \rho$. In turn, the Bohmian trajectories arising from the $\boldsymbol{v}^{K}$ streamlines can be measured by averaging over large numbers of identical single-particle events [13, 33, 34].

The relation that $S$ holds with the kinetic and canonical density current is of particular interest to the present work. It can be easily shown that the topological charge may be associated to the canonical density current by

$$
S=\frac{m}{2 \pi \hbar} \oint_{\mathcal{C}} \boldsymbol{v}^{C} \cdot d \boldsymbol{l},
$$

where the velocity $\boldsymbol{v}^{C}=\boldsymbol{J}^{C} / \rho$ is the canonical version of the Bohmian velocity. As the canonical and kinetic currents only differ by a term proportional to the vector potential, the Bohmian velocity is also connected to the topological charge through the relation

$$
S=\frac{m}{2 \pi \hbar} \oint_{\mathcal{C}} \boldsymbol{v}^{K} \cdot d \boldsymbol{l}-\frac{e}{2 \pi \hbar} \Phi_{B} .
$$

In the previous expression, $\Phi_{B}$ is the magnetic flux passing through the surface enclosed by $\mathcal{C}$. If $\mathcal{C}$ is very close to the singularity $\Phi_{B} \approx 0$ and the topological charge can be estimated entirely from the closed integral of $\boldsymbol{v}^{K}$.

Close to a singularity of the form (31) the canonical current components take the form

$$
\begin{aligned}
& J_{x}^{C}=-S|\gamma \beta||a|^{2} \frac{\hbar}{m}\left|\bar{z}-\bar{z}_{j}\right|^{2(s-1)}\left(y-y_{j}\right), \\
& J_{y}^{C}=S|\gamma \beta||a|^{2} \frac{\hbar}{m}\left|\bar{z}-\bar{z}_{j}\right|^{2(s-1)}\left(x-x_{j}\right) .
\end{aligned}
$$

From these expressions, it is clear that the canonical current close to the singularity spins in the direction indicated by the topological charge. Therefore, positive and negative topological charges yield counterclockwise and clockwise currents respectively whereas a vanishing topological charge yields a vanishing canonical current. Moreover, the topological charge can be determined from the 
shape of the Bohmian stream lines. By inserting the vector potential (2) in (36) the kinetic current components yield

$$
\begin{aligned}
J_{x}^{K}= & -\frac{|a|^{2}}{m}\left|\bar{z}-\bar{z}_{j}\right|^{2(s-1)} \\
& \times\left[S \hbar|\gamma \beta|\left(y-y_{j}\right)+\left|\bar{z}-\bar{z}_{j}\right|^{2} \frac{e B y}{2}\right], \\
J_{y}^{K}= & \frac{|a|^{2}}{m}\left|\bar{z}-\bar{z}_{j}\right|^{2(s-1)} \\
& \times\left[S \hbar|\gamma \beta|\left(x-x_{j}\right)+\left|\bar{z}-\bar{z}_{j}\right|^{2} \frac{e B x}{2}\right] .
\end{aligned}
$$

The components of the Bohmian velocity near the singularity are therefore

$$
\begin{aligned}
v_{x}^{K}= & -\frac{1}{m\left|\bar{z}-\bar{z}_{j}\right|^{2}} \\
& \times\left[S \hbar|\gamma \beta|\left(y-y_{j}\right)+\left|\bar{z}-\bar{z}_{j}\right|^{2} \frac{e B y}{2}\right], \\
v_{y}^{K}= & \frac{1}{m\left|\bar{z}-\bar{z}_{j}\right|^{2}} \\
& \times\left[S \hbar|\gamma \beta|\left(x-x_{j}\right)+\left|\bar{z}-\bar{z}_{j}\right|^{2} \frac{e B x}{2}\right] .
\end{aligned}
$$

The last four equations show that close to the singularity the direction of the kinetic density current is also determined by the the sign of the topological charge. Nevertheless the kinetic current might change direction away from the singularity when $S<0$. This is a characteristic feature of singularities having the opposite topological charge sign compared to the classical angular momentum of the electron. As we discuss below, this is exactly true for LG beams. It is worthwhile to notice that close to a singularity $\left(\left|\bar{z}-\bar{z}_{j}\right| \approx 0\right)$ the dominant contribution comes from the term proportional to $S$. Therefore the kinetic and the canonical currents as well as the velocities are similar, i.e. $\boldsymbol{J}^{K} \approx \boldsymbol{J}^{C}$ and

$$
\begin{aligned}
& v_{x}^{K} \approx v_{x}^{C}=-\frac{S \hbar|\gamma \beta|}{m\left|\bar{z}-\bar{z}_{j}\right|^{2}}\left(y-y_{j}\right), \\
& v_{y}^{K} \approx v_{y}^{C}=\frac{S \hbar|\gamma \beta|}{m\left|\bar{z}-\bar{z}_{j}\right|^{2}}\left(x-x_{j}\right) .
\end{aligned}
$$

In order to prove these results for the particular case of BEC's and HG beams we express the canonical and kinetic currents in terms of the LG beams. Calculating the explicit form of the velocity operators in terms of the rising and lowering operators (4)-(7) we obtain

$$
\begin{aligned}
& \hat{v}_{x}=\frac{\partial \hat{H}}{\partial \hat{p}_{x}}=\frac{1}{m}\left(\hat{p}_{x}-\frac{m \omega}{2} \hat{y}\right)=\frac{i \omega l_{B}}{\sqrt{2}}\left(\hat{c}^{\dagger}-\hat{c}\right), \\
& \hat{v}_{y}=\frac{\partial \hat{H}}{\partial \hat{p}_{y}}=\frac{1}{m}\left(\hat{p}_{y}+\frac{m \omega}{2} \hat{x}\right)=\frac{\omega l_{B}}{\sqrt{2}}\left(\hat{c}^{\dagger}+\hat{c}\right) .
\end{aligned}
$$

Similarly, for the momentum we have

$$
\begin{aligned}
& \hat{p}_{x}=i \frac{m \omega l_{B}}{2 \sqrt{2}}\left(\hat{c}^{\dagger}-\hat{c}+\hat{b}^{\dagger}-\hat{b}\right), \\
& \hat{p}_{y}=\frac{m \omega l_{B}}{2 \sqrt{2}}\left(\hat{c}^{\dagger}+\hat{c}-\hat{b}^{\dagger}-\hat{b}\right) .
\end{aligned}
$$

In order to get an insight on the behaviour of these currents in a simple case let us first calculate them for pure LG beams. Using the generalized Laguerre polynomials' recurrence relation

$$
L_{k+1}^{j-1}(x)=\frac{j-x}{k+1} L_{k}^{j}(x)-\frac{x}{k+1} L_{k-1}^{j+1}(x),
$$

we obtain the kinetic density currents components for LG beams

$$
\begin{aligned}
J_{x}^{L G, K}= & \operatorname{Re}\left[\frac{i \omega l_{B}}{\sqrt{2}} \psi_{l, n}^{L G *}(x, y)\left\langle x, y\left|\hat{c}^{\dagger}-\hat{c}\right| l, n\right\rangle\right] \\
& =-\rho_{l, n}^{L G}(x, y) \frac{\omega l_{B}^{2}}{r}\left(l-n+\frac{r^{2}}{2 l_{B}^{2}}\right) \sin \theta,(53) \\
J_{y}^{L G, K}= & \operatorname{Re}\left[\frac{\omega l_{B}}{\sqrt{2}} \psi_{l, n}^{L G *}(x, y)\left\langle x, y\left|\hat{c}^{\dagger}+\hat{c}\right| l, n\right\rangle\right] \\
& =\rho_{l, n}^{L G}(x, y) \frac{\omega l_{B}^{2}}{r}\left(l-n+\frac{r^{2}}{2 l_{B}^{2}}\right) \cos \theta, \quad(54)
\end{aligned}
$$

where $r=\sqrt{x^{2}+y^{2}}$ and $\theta=\arctan (y / x)$. These relations are consistent with the results presented in Ref. [30]. Replacing $\hat{p}_{x} / m=\hat{v}_{x}+\omega \hat{y} / 2$ and $\hat{p}_{y} / m=\hat{v}_{y}-\omega \hat{x} / 2$ into the kinetic density current components, the canonical ones are readily obtained

$$
\begin{aligned}
J_{x}^{L G, C}= & \operatorname{Re}\left[\psi_{l, n}^{*}(x, y)\right. \\
& \left.\times\left\langle x, y\left|i \frac{m \omega l_{B}}{\sqrt{2}}\left(\hat{c}^{\dagger}-\hat{c}\right)+\frac{\omega}{2} \hat{y}\right| l, n\right\rangle\right] \\
= & -\rho_{l, n}^{L G}(r) \frac{\omega l_{B}^{2}}{r}(l-n) \sin \theta, \\
J_{y}^{L G, C}= & \operatorname{Re}\left[\psi_{l, n}^{*}(x, y)\right. \\
& \left.\times\left\langle x, y\left|\frac{m \omega l_{B}}{\sqrt{2}}\left(\hat{c}^{\dagger}+\hat{c}\right)-\frac{\omega}{2} \hat{x}\right| l, n\right\rangle\right] \\
= & \rho_{l, n}^{L G}(r) \frac{\omega l_{B}^{2}}{r}(l-n) \cos \theta .
\end{aligned}
$$

These equations clearly show that both kinetic and canonical density currents similarly circulate around the singularity located at $z=0$. They spin in clockwise or counterclockwise direction depending on the sign of the angular momentum or topological charge $S=l-n$. This is a confirmation of the rather general results obtained in Eqs. (40)-(43). Indeed, it has been shown that for $m=-1$, the canonical density current spins around the singularity in counterclockwise direction, for $m=1$ it spins in clockwise direction and for $m=0$ the canonical current vanishes[15]. The kinetic density current, however, exhibits a more complicated behaviour. Whereas the canonical density current spins 
only in the direction indicated by the topological charge $S$, the kinetic density current changes direction in the orbit $r=l_{B} \sqrt{2(n-l)}$ provided that the angular momentum is negative $(l-n<0)$. This orbit corresponds precisely to the classical trajectory of the electron.

All of these features are clearly illustrated by plotting the path integrals

$$
\begin{aligned}
\eta^{K} & =\oint_{\mathcal{C}} \boldsymbol{J}^{K} \cdot d \boldsymbol{l}, \\
\eta^{C} & =\oint_{\mathcal{C}} \boldsymbol{J}^{C} \cdot d \boldsymbol{l},
\end{aligned}
$$

where $\mathcal{C}$ is a circle of radius $r$. These two gauge invariant functions differ only by a multiple of the Dirac phase.

The gain of using these two functions becomes evident when we calculate them in the proximity of a singularity of the type (31) obtaining

$$
\begin{aligned}
\eta^{C} & =2 \pi \omega l_{B}^{2} S \rho(r), \\
\eta^{K} & =2 \pi \omega l_{B}^{2}\left(S+\frac{\gamma+\beta}{2 \gamma \beta} \frac{r^{2}}{2 l_{B}^{2}}\right) \rho(r),
\end{aligned}
$$

where $\rho(r)=|a|^{2} r^{2 s}$ is the probability density close to the singularity $z_{j}$. These equations imply that the topological charge can be computed analytically by taking the limit as $z$ approaches $z_{j}$ of either $\eta^{K} / 2 \pi \omega l_{B}^{2} \rho$ or $\eta^{C} / 2 \pi \omega l_{B}^{2} \rho$. The functions $\eta^{K}$ and $\eta^{C}$ are easier to calculate than the actual phase change around a singularity $\int d \varphi$ that in many occasions can only be computed numerically.

The particular case of LG beams is quite illustrative in this matter. LG beams yield

$$
\begin{aligned}
\eta^{K} & =2 \pi \omega l_{B}^{2}\left(l-n+\frac{r^{2}}{2 l_{B}^{2}}\right) \rho_{l, n}^{L G}(r), \\
\eta^{C} & =2 \pi \omega l_{B}^{2}(l-n) \rho_{l, n}^{L G}(r) .
\end{aligned}
$$

Notice that (59) and (60) reduce to the expressions above provided that we set $S=l-n$ and the integral is performed around a circle, namely $\gamma=\beta=1$.

From the previous equations we observe that while $\eta^{K}>0$ for $m=0,1$ for all $r$ values, $\eta^{K}>0$ with $m=-1$ presents a sign change at $r=\sqrt{2} l_{B}$ characteristic of diamagnetic states. The expression of $\eta^{C}$ clearly shows that the canonical currents spin in the expected direction given by the sign of the angular momentum and the topological charge $S$. By taking the limit $r \rightarrow 0$ of $\eta^{C} / 2 \pi \omega l_{B}^{2} \rho$ or $\eta^{K} / 2 \pi \omega l_{B}^{2} \rho$ we obtain the topological charge $S$ confirming the result in Eqs. (59) and (60).

By inserting the velocity operators (48) and (49) into the definition of the density current (37) for a general state of the form (13) we get

$$
\begin{aligned}
J_{x}^{K}= & \operatorname{Re}\left[i \frac{\omega l_{B}}{\sqrt{2}} \sum_{l, l^{\prime}, n, n^{\prime}} A_{l^{\prime}, n^{\prime}}^{*} A_{l, n} \mathrm{e}^{i \omega\left(l^{\prime}-l\right) t} \psi_{l^{\prime}, n^{\prime}}^{L G *}\right. \\
& \left.\times\left(\sqrt{l+1} \psi_{l+1, n}^{L G}-\sqrt{l} \psi_{l-1, n}^{L G}\right)\right], \\
J_{y}^{K}= & \operatorname{Re}\left[\frac{\omega l_{B}}{\sqrt{2}} \sum_{q, p=q_{\min }}^{q_{\max }} A_{p}^{j, k} A_{q}^{j, k} \mathrm{e}^{i \omega\left(l^{\prime}-l\right) t} \psi_{l^{\prime}, n^{\prime}}^{L G *}\right. \\
& \left.\times\left(\sqrt{l+1} \psi_{l+1, n}^{L G}+\sqrt{l} \psi_{l-1, n}^{L G}\right)\right] .
\end{aligned}
$$

Similarly, inserting the components of the momentum operator (50)-(51) into (36) the canonical current is readily obtained as

$$
\begin{aligned}
J_{x}^{C}= & \operatorname{Re}\left[i \frac{\omega l_{B}}{2 \sqrt{2}} \sum_{l, l^{\prime}, n, n^{\prime}} A_{l^{\prime}, n^{\prime}}^{*} A_{l, n} \mathrm{e}^{i \omega\left(l^{\prime}-l\right) t} \psi_{l^{\prime}, n^{\prime}}^{L G *}\right. \\
& \times\left(\sqrt{l+1} \psi_{l+1, n}^{L G}-\sqrt{l} \psi_{l-1, n}^{L G}\right. \\
& \left.\left.+\sqrt{n+1} \psi_{l, n+1}^{L G}-\sqrt{n} \psi_{l, n-1}^{L G}\right)\right], \\
J_{y}^{C}= & \operatorname{Re}\left[\frac{\omega l_{B}}{2 \sqrt{2}} \sum_{l, l^{\prime}, n, n^{\prime}} A_{l^{\prime}, n^{\prime}}^{*} A_{l, n} \mathrm{e}^{i \omega\left(l^{\prime}-l\right) t} \psi_{l^{\prime}, n^{\prime}}^{L G *}\right. \\
& \times\left(\sqrt{l+1} \psi_{l+1, n}^{L G}+\sqrt{l} \psi_{l-1, n}^{L G}\right. \\
& \left.\left.-\sqrt{n+1} \psi_{l, n+1}^{L G}-\sqrt{n} \psi_{l, n-1}^{L G}\right)\right] .
\end{aligned}
$$

In the particular case of $\mathrm{HG}$ beams the kinetic density current is given by

$$
\begin{aligned}
J_{x}^{H G, K} & =\operatorname{Re}\left[i \frac{\omega l_{B}}{\sqrt{2}} \sum_{q, p=q_{\min }}^{q_{\max }}\left(A_{p}^{j, k}\right)^{*} A_{q}^{j, k} \mathrm{e}^{i \omega(p-q) t} \psi_{p, j+k-p}^{*}\right. \\
& \left.\times\left(\sqrt{q+1} \psi_{q+1, j+k-q}-\sqrt{q} \psi_{q-1, j+k-q}\right)\right], \quad(67) \\
J_{y}^{H G, K} & =\operatorname{Re}\left[\frac{\omega l_{B}}{\sqrt{2}} \sum_{q, p=q_{\min }}^{q_{\max }}\left(A_{p}^{j, k}\right)^{*} A_{q}^{j, k} \mathrm{e}^{i \omega(p-q) t} \psi_{p, j+k-p}^{*}\right. \\
& \left.\times\left(\sqrt{q+1} \psi_{q+1, j+k-q}+\sqrt{q} \psi_{q-1, j+k-q}\right)\right] .
\end{aligned}
$$


For the canonical density current of HG beams we have

$$
\begin{aligned}
J_{x}^{H G, C}= & \operatorname{Re}\left[i \frac{\omega l_{B}}{2 \sqrt{2}} \sum_{q, p=q_{\min }}^{q_{\max }}\left(A_{p}^{j, k}\right)^{*} A_{q}^{j, k} \mathrm{e}^{i \omega(p-q) t} \psi_{p, j+k-p}^{*}\right. \\
& \times\left(\sqrt{q+1} \psi_{q+1, j+k-q}-\sqrt{q} \psi_{q-1, j+k-q}\right. \\
& +\sqrt{j+k-q+1} \psi_{q, j+k-q+1} \\
& \left.\left.-\sqrt{j+k-q-1} \psi_{q, j+k-q-1}\right)\right] \\
J_{y}^{H G, C}= & \operatorname{Re}\left[\frac{\omega l_{B}}{2 \sqrt{2}} \sum_{q, p=q_{\min }}^{q_{\max }}\left(A_{p}^{j, k}\right)^{*} A_{q}^{j, k} \mathrm{e}^{i \omega(p-q) t} \psi_{p, j+k-p}^{*}\right. \\
& \times\left(\sqrt{q+1} \psi_{q+1, j+k-q}+\sqrt{q} \psi_{q-1, j+k-q}\right. \\
& -\sqrt{j+k-q+1} \psi_{q, j+k-q+1} \\
& \left.\left.-\sqrt{j+k-q-1} \psi_{q, j+k-q-1}\right)\right]
\end{aligned}
$$

For standard HG beam, $q_{\min }=0$ and $q_{\max }=j+k$. Instead, for the positive and negative part of HG beams $q_{\min }$ and $q_{\max }$ are given by Eqs. (23)-(26). In the following sections, the expressions above will be very useful in exploring the vorticity of density currents around singularities.

\section{RESULTS AND DISCUSSION}

In this section we wish to prove the general relations between the singularities' mathematical structure and the kinetic and canonical currents in more involved electron beams. In order to analyse sufficiently complex systems with a rich singularity structure we have chosen HG and BEC beams.

We begin by investigating $\psi_{3,3}^{H G}$ and $\psi_{3,3}^{ \pm H G}$. The state $\psi_{3,3}^{H G}$ has the structure of a balanced beam given by

$$
\begin{aligned}
\psi_{3,3}^{H G}=-\frac{i}{4}\left(\sqrt{5} \psi_{0,6}^{L G}-\sqrt{3}\right. & \psi_{2,4}^{L G} \\
& \left.+\sqrt{3} \psi_{4,2}^{L G}-\sqrt{5} \psi_{6,0}^{L G}\right) .
\end{aligned}
$$

Substituting the explicit form of $\psi_{3,3}^{H G}$ into Eqs. (69) and (70) the canonical density current vanishes $\boldsymbol{J}_{3,3}^{H G, C}=\mathbf{0}$ in the whole domain of the wave function. Thereby, the kinetic density current $\boldsymbol{J}_{3,3}^{H G, K}=e B(-y \boldsymbol{i}+x \boldsymbol{j}) \rho_{3,3}^{H G} / m$, that only contains the terms arising from the vector potential, spins in the counterclockwise direction as it can be seen in Fig. 1. In contrast to LG beams, that have singularities in isolated points, HG beams present singular points arranged as lines [23]. In Fig. 1 (a) we show the structure of the $\psi_{3,3}^{H G}$ singularities. They can conveniently be grouped in one point [(purple) dot] and 6 lines (dashed lines) listed in rows 3-7 of Table I. Except for the point (row 3) all of these singularities can be put in the form (31) where $\gamma \beta=0$. Under the arguments presented in Secs. IV and V the canonical currents close to these singularities must vanish as expected. Figs. 1 (a), (b) and (c) also show the time evolution of the probability density $\rho_{3,3}^{H G}$ for times $t=0, t=\pi / 4 \omega$ and $t=\pi / 2 \omega$ respectively. Given that the beam is a superposition of states with different eigenenergies it presents a nontrivial time evolution. In this case, the singularity lines rotate in the counterclockwise direction, in the same direction a classical electron would move under the action of a perpendicular magnetic field. This behaviour is observed in all of the singularities presented from here on.

The singularities in states $\psi_{3,3}^{ \pm H G}$ have a completely different structure. They can be expressed in terms of the LG states as

$$
\begin{aligned}
& \psi_{3,3}^{-H G}=-\frac{i}{4}\left(\sqrt{5} \psi_{0,6}^{L G}-\sqrt{3} \psi_{2,4}^{L G}\right), \\
& \psi_{3,3}^{+H G}=-\frac{i}{4}\left(\sqrt{3} \psi_{4,2}^{L G}-\sqrt{5} \psi_{6,0}^{L G}\right) .
\end{aligned}
$$

The above states have a total of 13 singularities arranged as isolated points. These are shown in Figs. 2 (a) and 3 (a) as dots and are listed in rows 8-15 in Table I. A more detailed diagram of the four different types of singularities and their corresponding topological charges is shown in Fig. 4. The singularity located at the center $z=0$ has a topological charge $S_{3,3}^{ \pm}= \pm 2$. Along the lines forming angles of $0^{\circ}, 90^{\circ}, 180^{\circ}$ and $270^{\circ}$ we have two different types of singularities. The first type with $S_{3,3}^{ \pm}= \pm 1$ and the second with $S_{3,3}^{ \pm}=\mp 1$. Along lines forming angles of $45^{\circ}, 135^{\circ}, 225^{\circ}$ and $315^{\circ}$ with the $x$ axis we find the fourth kind of singularity having a vanishing topological charge $S_{3,3}^{ \pm}=0$. By using Eqs. (67), (68), (69) and (70) we can compute the components of the kinetic and canonical density currents. The streamlines of the $\psi_{3,3}^{+H G}$ kinetic density current, namely the Bohmian trajectories, are presented in Figs. 2 (a) and (b) while (c) and (d) show the canonical density current. Similarly, Figs. 3 (a) and (b) plot the kinetic density current and (c) and (d) the canonical one for $\psi_{3,3}^{-H G}$. We immediately notice that, in contrast to $\psi_{3,3}^{+H G}$, the streamlines in $\psi_{3,3}^{-H G}$ switch from clockwise to counterclockwise direction as we move away from the center of the beam. As it was discussed for LG beams, it is a distinctive feature of singularities having the opposite topological charge sign to the spinning direction of the classical electron.

The same vector fields close to the four types of singularities are shown in Figs. 5 and 6 for $\psi_{3,3}^{+H G}$ and $\psi_{3,3}^{-H G}$ respectively. Only the canonical current is shown in this case because, given the proximity to the singularities, the terms related to the vector potential do not strongly distort the general form of the current streamlines. In each case we confirm that the canonical current spins in the direction indicated by the topological charge.

Figs. 7 (a) and (b) show plots of $\eta^{K}$ and $\eta^{C}$ respectively. In this case they are calculated through Eqs. (57) and (58) integrating around a circle of radius $r=\sqrt{x^{2}+y^{2}}$. In these figures it is possible to note that the kinetic density current of the negative angular momentum part of $\psi_{3,3}^{H G}$ presents a sign change that is 
consistent with the relative sign of the singularity's topological charge compared to the angular momentum of the classical electron. This same feature was discussed above regarding the Bohmian trajectories observed in Figs. 3 and 4 .

Let us now turn our attention to the calculation of the topological charge via the limit $S=$ $\lim _{r \rightarrow 0} \eta^{K} / 2 \pi \omega l_{B}^{2} \rho=\lim _{r \rightarrow 0} \eta^{C} / 2 \pi \omega l_{B}^{2} \rho$. In Fig. 8 we have plotted $\eta^{K} / 2 \pi \omega l_{B}^{2} \rho$ and $\eta^{C} / 2 \pi \omega l_{B}^{2} \rho$ as functions of the distance to the singularity $r$ for the four types of singularities (shown in Figs. 5 and 6 ) found in the $\psi_{3,3}^{ \pm H G}$ beams. Figs. 8 (a), (b), (c) and (d) correspond to the singularities listed in Table I in rows 8-11 for $\psi_{3,3}^{-H G}$ and rows 12-15 for $\psi_{3,3}^{+H G}$. Given the high symmetry of $S_{3,3}^{ \pm H G}= \pm 2[$ Fig. $8(\mathrm{a})]$, the corresponding $\eta^{K} / 2 \pi \omega l_{B}^{2} \rho$ and $\eta^{C} / 2 \pi \omega l_{B}^{2} \rho$ where calculated exactly. However, in order to plot $\eta^{K} / 2 \pi \omega l_{B}^{2} \rho$ and $\eta^{C} / 2 \pi \omega l_{B}^{2} \rho$ for the three remaining singularities $S_{3,3}^{ \pm H G}= \pm 1, \mp 1,0$ with lower symmetry, a Taylor expansion of order 12 in $r$ around the singularity $(r=0)$ was used. Even though such high order expansions are needed to obtain accurate plots, the calculation of the topological charge only requires a Taylor expansion of the lowest non-vanishing order. For example, expanding $\eta^{C} / 2 \pi \omega l_{B}^{2} \rho$ to the first order in $r$ for $S_{3,3}^{-H G}=-1$ around $z=2 l_{B} \sqrt{3 /(2+\sqrt{2})}+i 0$ with $\gamma=1$ and $\beta=(\sqrt{2}-1) / 2$ (see Table I, row 9) we get

$$
\begin{aligned}
\eta^{C} / 2 \pi \omega l_{B}^{2} \rho \approx & -1+\frac{r}{12 l_{B}} \sqrt{3(2+\sqrt{2})} \cos \theta \\
& \times[10 \sqrt{2}-3-(7+2 \sqrt{2}) \cos 2 \theta] .
\end{aligned}
$$

Similarly for $S_{3,3}^{-H G}=1$, expanding around $z=$ $2 l_{B} \sqrt{3 /(2-\sqrt{2})}+i 0$ with $\gamma=1$ and $\beta=(1+\sqrt{2}) / 2$ (see Table I, row 10) we have

$$
\begin{aligned}
\eta^{C} / 2 \pi \omega l_{B}^{2} \rho \approx 1+\frac{r}{24 l_{B}} \sqrt{3(2+\sqrt{2})} \\
\quad \times[(7+2 \sqrt{2}) \cos 3 \theta-(18 \sqrt{2}-13) \cos \theta],
\end{aligned}
$$

and for $S_{3,3}^{-H G}=0$, expanding around $z=\sqrt{3}+i \sqrt{3}$ with $\gamma=1$ and $\beta=0$ (see Table I, row 10) we have

$$
\eta^{C} / 2 \pi \omega l_{B}^{2} \rho=0 .
$$

The three functions clearly reduce to their corresponding topological charges when the limit $r \rightarrow 0$ is taken.

To further prove the formulas obtained in Sec. $\mathrm{V}$ we also study BEC states. These have $n$ singularities arranged as isolated and equally spaced points. In Fig. 9 we illustrate the time evolution of the $\psi_{3}^{+B E C}$ beam. The vector field represents the $\boldsymbol{J}^{K}$ density currents in (a) and (b) and $\boldsymbol{J}^{C}$ in (c) and (d). Fig. 10 shows the time evolution of the $\psi_{3}^{-B E C}$ beam. We observe again the features seen for LG and HG beams: i) in the positive beam $\psi_{3}^{+B E C}$ both kinetic and canonical currents spin in counterclockwise direction, ii) in the negative beam $\psi_{3}^{-B E C}$ the kinetic current near the singularities spin in the clockwise direction and iii) as we move away from the singularities the counterclockwise direction of the kinetic current is recovered. The positive beam $\psi_{3}^{+B E C}$ is not an energy eigenstate and therefore it rotates in the counterclockwise direction as it evolves in time. The negative beam, however, does not rotate since it is a stationary state, composed of two LG beams with the same energy $\hbar \omega / 2(l=0)$.

The behaviour of the kinetic currents close to the singularities is shown in Fig. 11 for (a) $\psi_{3}^{+B E C}$ and (b) $\psi_{3}^{-B E C}$ (see row 16 in Table I ). We observe that the direction of the kinetic current is determined again by the sign of the topological charge.

Now, expanding $\eta^{C} / 2 \pi \omega l_{B}^{2} \rho$ and $\eta^{K} / 2 \pi \omega l_{B}^{2} \rho$ to the first order, we obtain the $\psi_{3}^{ \pm B E C}$ topological charges. For $S_{3}^{-B E C}=-1$ we have $\gamma=1$ and $\beta=-1$ (see row 16 in Table I)

$$
\begin{aligned}
& \eta^{C} / 2 \pi \omega l_{B}^{2} \rho \approx \eta^{K} / 2 \pi \omega l_{B}^{2} \rho \approx-1+\frac{r}{\sqrt{3} l_{B}}\left(\frac{3}{2}\right)^{1 / 3} \\
& \times\left[1-\left(\frac{3}{2}\right)^{1 / 3}\right]\left(\frac{1}{\sqrt{3}} \cos \theta+\sin \theta\right) .
\end{aligned}
$$

Similarly the singularity $S_{3}^{+B E C}=1$ we have $\gamma=1$ and $\beta=1$ (see row 17 in Table I)

$$
\begin{aligned}
& \eta^{C} / 2 \pi \omega l_{B}^{2} \rho \approx \eta^{K} / 2 \pi \omega l_{B}^{2} \rho \approx 1-\frac{r}{\sqrt{3} l_{B}}\left(\frac{3}{2}\right)^{1 / 3} \\
& \times\left[1-\left(\frac{3}{2}\right)^{1 / 3}\right]\left(\frac{1}{\sqrt{3}} \cos \theta+\sin \theta\right) .
\end{aligned}
$$

Again we observe that the limit of the expressions above as the distance to the singularity approaches zero is equal to the topological charge.

\section{FINDING THE MATHEMATICAL STRUCTURE OF A SINGULARITY FROM THE BOHMIAN STREAMLINES}

Using the previous relations, in this section we outline a method to obtain the mathematical structure of the wave function near a singularity. Starting from the Bohmian streamlines, we work backward obtaining the main parameters that define the wave function.

As an example, let us consider the beam characterized 
by the wave function

$$
\begin{aligned}
\psi(x, y)= & \frac{1}{\mathcal{N} l_{B}^{4}} \exp \left(-\frac{x^{2}+y^{2}}{4 l_{B}^{2}}\right) \\
& \times\left\{\left[\frac{\sqrt{3}}{2}\left(x-3 l_{B}\right)+\frac{1}{2}\left(y-l_{B}\right)\right]\right. \\
& \left.+i \frac{3}{2}\left[\frac{\sqrt{3}}{2}\left(y-l_{B}\right)-\frac{1}{2}\left(x-3 l_{B}\right)\right]\right\}^{3},
\end{aligned}
$$

where $\mathcal{N}=\sqrt{(6192176-1960965 \sqrt{3}) \pi} / 16$ is a normalisation constant. From the structure of the wave function it is easy to show that it only has one singularity located in $x_{j}=3 l_{B}$ and $y_{j}=l_{b}$, with $S=+3, \gamma=1, \beta=3 / 2$ and it is tilted $30^{\circ}$ with respect to the $x$-axis. The wave function (79) can be expressed in terms of the LG modes as

$$
\begin{array}{r}
\psi=A_{0,0} \psi_{0,0}^{L G}+A_{0,1} \psi_{0,1}^{L G}+A_{0,2} \psi_{0,2}^{L G}+A_{0,3} \psi_{0,3}^{L G} \\
+A_{1,0} \psi_{1,0}^{L G}+A_{1,1} \psi_{1,1}^{L G}+A_{1,2} \psi_{1,2}^{L G}+A_{2,0} \psi_{2,0}^{L G} \\
+A_{2,1} \psi_{2,1}^{L G}+A_{3,0} \psi_{3,0}^{L G}
\end{array}
$$

where the coefficients are given by

$$
\begin{aligned}
& A_{0,0}=\frac{1}{\mathcal{N}}\left(\frac{1}{4}-\frac{3 i}{16}\right) \\
& \times[(-247-138 i)+(63+126 i) \sqrt{3}] \sqrt{\frac{\pi}{2}}, \\
& A_{0,1}=-\frac{1}{\mathcal{N}}\left(\frac{27}{32}+\frac{3 i}{16}\right) \\
& \times[(5-18 i)+(6+7 i) \sqrt{3}] \sqrt{\pi}, \\
& A_{0,2}=-\frac{1}{\mathcal{N}}\left(\frac{9}{32}-\frac{3 i}{32}\right) \\
& \times[(-3+2 i)+(4+3 i) \sqrt{3}] \sqrt{\pi}, \\
& A_{0,3}=-\frac{1}{8 \mathcal{N}} i \sqrt{\frac{3 \pi}{2}} \\
& A_{1,0}=-\frac{1}{\mathcal{N}}\left(\frac{15}{16}-\frac{135 i}{32}\right) \\
& \times[(-18-13 i)+(1+6 i) \sqrt{3}] \sqrt{\pi}, \\
& A_{1,1}=\frac{1}{\mathcal{N}}\left(\frac{15}{4}+\frac{15 i}{8}\right)[3 \sqrt{3}-(1+4 i)] \sqrt{\frac{\pi}{2}}, \\
& A_{1,2}=\frac{15}{16 \mathcal{N}}(\sqrt{3}+i) \sqrt{\frac{\pi}{2}} \text {, } \\
& A_{2,0}=\frac{1}{\mathcal{N}}\left(\frac{75}{32}+\frac{225 i}{32}\right)[(7+6 i)-i \sqrt{3}] \sqrt{\pi}, \\
& A_{2,1}=\frac{75}{16 \mathcal{N}}(\sqrt{3}-i) \sqrt{\frac{\pi}{2}} \\
& A_{3,0}=-\frac{125}{8 \mathcal{N}} i \sqrt{\frac{3 \pi}{2}} \text {. }
\end{aligned}
$$
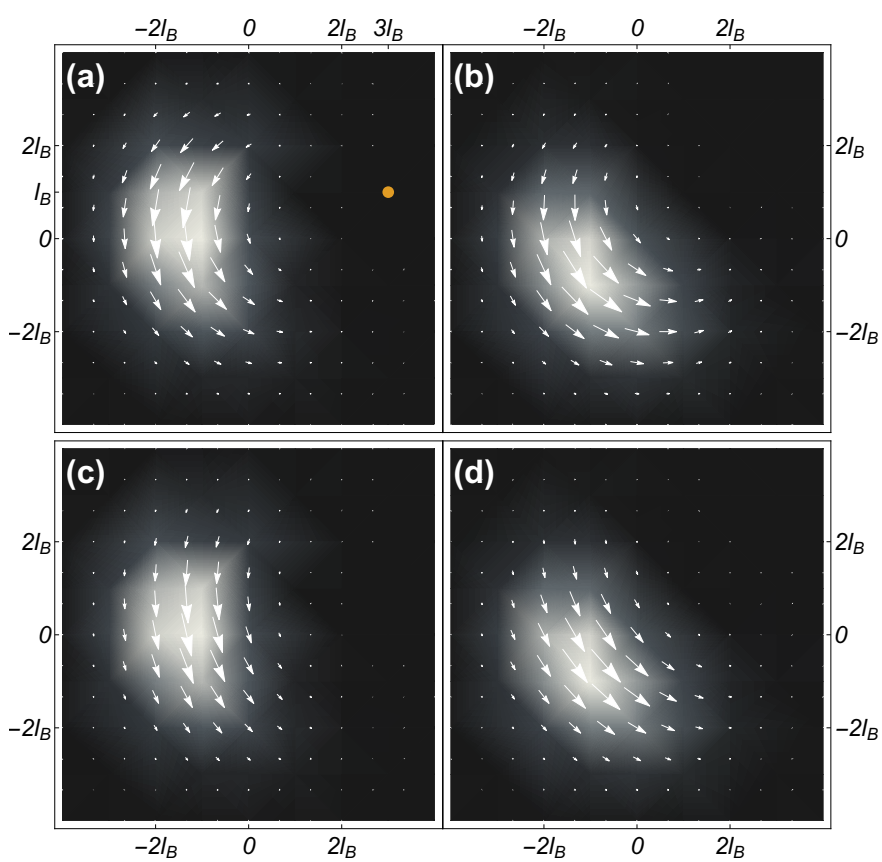

FIG. 14. Rotational dynamics of $\psi$. Along the first row, in (a) and (b) we observe the time evolution of $\rho=|\psi|^{2}$ and the vector field $\boldsymbol{J}^{K}$ for $t=0$ and $t=\pi / 6 \omega$ respectively. In the second row, (c) and (d) show the time evolution of $\rho$ and the vector field $\boldsymbol{J}^{C}$ for $t=0$ and $t=\pi / 6 \omega$ respectively. The positions of the singularity $z_{j}=3 l_{B}+i l_{B}$ is indicated with an (orange) dot in (a).

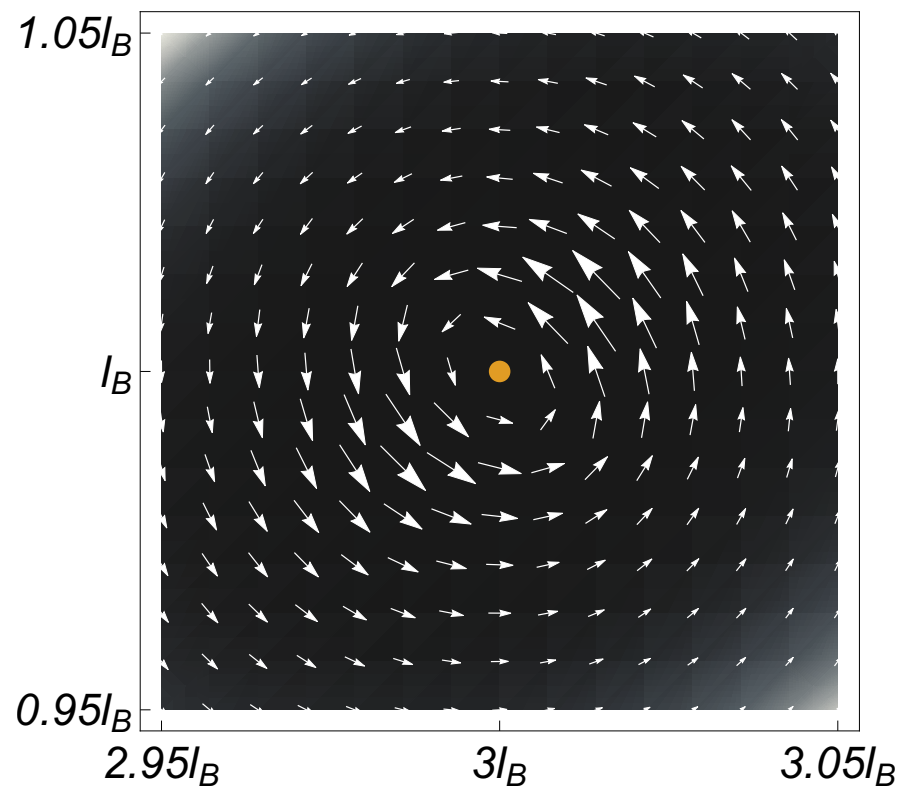

FIG. 15. Bohmian velocity around the singularitiy $z_{j}=3 l_{B}+$ $i l_{B}$ of $\psi$. The position of the singularity is indicated with a dot. The density plot of the density probability $\rho=|\psi|^{2}$ and the corresponding Bohmian velocity field $\boldsymbol{v}^{K}$ are also shown. 
TABLE I. List of singularities. The first column presents the state

\begin{tabular}{|c|c|c|c|c|}
\hline$\psi$ & $z_{j}$ & $S$ & $\lim _{z \rightarrow z_{j}} \psi$ & $\gamma \beta$ \\
\hline $\begin{array}{l}\psi_{l, n}^{L G} \\
n>l\end{array}$ & 0 & $S_{l, n}^{L G}=l-n$ & $\frac{l !}{\pi n !\left(2 l_{B}^{2}\right)^{n-l}} L_{l}^{n-l}(0)\left[\left(x-x_{j}\right)-i\left(y-y_{j}\right)\right]^{n-l}$ & $\gamma \beta<0$ \\
\hline $\begin{array}{l}\psi_{l, n}^{L G} \\
l>n\end{array}$ & 0 & $S_{l, n}^{L G}=l-n$ & $\frac{n !}{\pi l !\left(2 l_{B}^{2}\right)^{l-n}} L_{n}^{l-n}(0)\left[\left(x-x_{j}\right)+i\left(y-y_{j}\right)\right]^{l-n}$ & $\gamma \beta>0$ \\
\hline$\psi_{3,3}^{H G}$ & $0+i 0$ & $S_{3,3}^{H G}=0$ & $\frac{3}{2 l_{B}^{3} \sqrt{2 \pi}}\left(x-x_{j}\right)\left(y-y_{j}\right)$ & $\gamma \beta=0$ \\
\hline$\psi_{3,3}^{H G}$ & $0+i d, d \neq 0$ & $S_{3,3}^{H G}=0$ & $-\frac{d\left(d^{2}-3 l_{B}^{2}\right)}{2 l_{B}^{5} \sqrt{2 \pi}} \mathrm{e}^{-\frac{d^{2}}{4 l_{B}^{2}}}\left(x-x_{j}\right)$ & $\gamma \beta=0$ \\
\hline$\psi_{3,3}^{H G}$ & $d+i 0, d \neq 0$ & $S_{3,3}^{H G}=0$ & $-\frac{d\left(d^{2}-3 l_{B}^{2}\right)}{2 l_{B}^{5} \sqrt{2 \pi}} \mathrm{e}^{-\frac{d^{2}}{4 l_{B}^{2}}}\left(y-y_{j}\right)$ & $\gamma \beta=0$ \\
\hline$\psi_{3,3}^{H G}$ & $\pm \sqrt{3} l_{B}+i d$ & $S_{3,3}^{H G}=0$ & $-\frac{d\left(d^{2}-3 l_{B}^{2}\right)}{2 l_{B}^{5} \sqrt{2 \pi}} \mathrm{e}^{-\frac{d^{2}}{4 l_{B}^{2}-\frac{9}{4}}}\left\{\left(x-x_{j}\right) \mp 3\left[\frac{2 l_{B}}{d}-\frac{d}{l_{B}}\left(1-\frac{4 l_{B}^{2}}{d^{2}-3 l_{B}^{2}}\right)\right]\left(y-y_{j}\right)\right\}$ & $\gamma \beta=0$ \\
\hline$\psi_{3,3}^{H G}$ & $d \pm i \sqrt{3} l_{B}$ & $S_{3,3}^{H G}=0$ & $-\frac{d\left(d^{2}-3 l_{B}^{2}\right)}{2 l_{B}^{5} \sqrt{2 \pi}} \mathrm{e}^{-\frac{d^{2}}{4 l_{B}^{2}-\frac{9}{4}}}\left\{\mp 3\left[\frac{2 l_{B}}{d}-\frac{d}{l_{B}}\left(1-\frac{4 l_{B}^{2}}{d^{2}-3 l_{B}^{2}}\right)\right]\left(x-x_{j}\right)\left(y-y_{j}\right)\right\}$ & $\gamma \beta=0$ \\
\hline$\psi_{3,3}^{-H G}$ & 0 & $S_{3,3}^{-H G}=-2$ & $\frac{3 i}{8 \sqrt{2 \pi} l_{B}^{3}}\left[\left(x-x_{j}\right)-i\left(y-y_{j}\right)\right]^{2}$ & $\gamma \beta<0$ \\
\hline$\psi_{3,3}^{-H G}$ & $\begin{array}{l}2 l_{B} \sqrt{\frac{3}{2+\sqrt{2}}} \mathrm{e}^{i n \pi / 4} \\
\quad n=0,2,4,6\end{array}$ & $S_{3,3}^{-H G}=-1$ & $-\frac{3}{2 l_{B}^{2}} i \mathrm{e}^{\frac{3}{\sqrt{2}}-3} \sqrt{\frac{3(10-7 \sqrt{2})}{\pi}}\left[\left(x-x_{j}\right)+\frac{i}{2}(1-\sqrt{2})\left(y-y_{j}\right)\right]$ & $\gamma \beta<0$ \\
\hline$\psi_{3,3}^{-H G}$ & $\begin{array}{c}2 l_{B} \sqrt{\frac{3}{2-\sqrt{2}}} \mathrm{e}^{i n \pi / 4}, \\
n=0,2,4,6\end{array}$ & $S_{3,3}^{-H G}=+1$ & $\frac{3}{2 l_{B}^{2}} i \mathrm{e}^{-3-\frac{3}{\sqrt{2}}} \sqrt{\frac{3(10+7 \sqrt{2})}{\pi}}\left[\left(x-x_{j}\right)+\frac{i}{2}(1+\sqrt{2})\left(y-y_{j}\right)\right]$ & $\gamma \beta>0$ \\
\hline$\psi_{3,3}^{-H G}$ & $\begin{array}{l}\sqrt{6} l_{B} \mathrm{e}^{i n \pi / 4} \\
\quad n=1,3,5,7\end{array}$ & $S_{3,3}^{-H G}=0$ & $\frac{3 i \sqrt{\frac{3}{2 \pi}}}{8 l_{B}^{2} \mathrm{e}^{3 / 2}}\left[\left(x-x_{j}\right)-\left(y-y_{j}\right)\right]$ & $\gamma \beta=0$ \\
\hline$\psi_{3,3}^{+H G}$ & 0 & $S_{3,3}^{+H G}=+2$ & $-\frac{3 i}{8 l_{B}^{3} \sqrt{2 \pi}}\left[\left(x-x_{j}\right)+i\left(y-y_{j}\right)\right]^{2}$ & \\
\hline$\psi_{3,3}^{+H G}$ & $\begin{array}{l}2 l_{B} \sqrt{\frac{3}{2+\sqrt{2}}}, \mathrm{e}^{i n \pi / 4} \\
n=0,2,4,6\end{array}$ & $S_{3,3}^{+H G}=+1$ & $\frac{3}{2 l_{B}^{2}} i \mathrm{e}^{\frac{3}{\sqrt{2}}-3} \sqrt{\frac{3(10-7 \sqrt{2})}{\pi}}\left[\left(x-x_{j}\right)-\frac{i}{2}(1-\sqrt{2})\left(y-y_{j}\right)\right]$ & $\gamma \beta>0$ \\
\hline$\psi_{3,3}^{+H G}$ & $\begin{array}{c}2 l_{B} \sqrt{\frac{3}{2-\sqrt{2}}} \mathrm{e}^{i n \pi / 4}, \\
n=0,2,4,6\end{array}$ & $S_{3,3}^{+H G}=-1$ & $-\frac{3}{2 l_{B}^{2}} i \mathrm{e}^{-3-\frac{3}{\sqrt{2}}} \sqrt{\frac{3(10+7 \sqrt{2})}{\pi}}\left[\left(x-x_{j}\right)-\frac{i}{2}(1+\sqrt{2})\left(y-y_{j}\right)\right]$ & $\gamma \beta<0$ \\
\hline$\psi_{3,3}^{+H G}$ & $\begin{array}{l}\sqrt{6} l_{B} \mathrm{e}^{i n \pi / 4} \\
\quad n=1,3,5,7 \\
\end{array}$ & $S_{3,3}^{+H G}=0$ & $-\frac{3 i \sqrt{\frac{3}{2 \pi}}}{8 l_{B}^{2} \mathrm{e}^{3 / 2}}\left[\left(x-x_{j}\right)-\left(y-y_{j}\right)\right]$ & $\gamma \beta=0$ \\
\hline$\psi_{3}^{-B E C}$ & $\begin{array}{l}\sqrt[3]{2 \sqrt{3}} l_{B} \mathrm{e}^{i n \pi / 3} \\
n=1,3,5\end{array}$ & $S_{3}^{-B E C}=-1$ & $-\frac{\sqrt[3]{3}(\sqrt{3}+3 i) \mathrm{e}^{-\frac{1}{2}} \sqrt[3]{\frac{3}{2}}}{2^{11 / 6} l_{B}^{2} \sqrt{5 \pi}}\left[\left(x-x_{j}\right)-i\left(y-y_{j}\right)\right]$ & $\gamma \beta<0$ \\
\hline$\psi_{3}^{+B E C}$ & $\begin{array}{l}\sqrt[3]{2 \sqrt{3}} l_{B} \mathrm{e}^{i n \pi / 3} \\
n=1,3,5\end{array}$ & $S_{3}^{+B E C}=+1$ & $-\frac{\sqrt[3]{3}(\sqrt{3}+3 i) \mathrm{e}^{-\frac{1}{2}} \sqrt[3]{\frac{3}{2}}}{2^{11 / 6} l_{B}^{2} \sqrt{5 \pi}}\left[\left(x-x_{j}\right)+i\left(y-y_{j}\right)\right]$ & $\gamma \beta>0$ \\
\hline
\end{tabular}

Using Eqs. (63)-(66) we calculate the kinetic and canonical density currents shown in Fig. 14. Notice that the probability density evolves by rotating in the counterclockwise direction. In the same figure we also show the position of the singularity whose wave function we wish to characterize.

The starting point is a grid of $16 \times 16$ nodes centered in the singularity. In each one of them the values of the $x$ and $y$ components of the Bohmian velocity are known. We have intentionally added a $10 \%$ error to the velocity components. A zoom of the Bohmian velocity field of $\psi$ close to the singularity is exhibited in Fig. 15.

The topological charge is the first parameter that we extract from this map. Given that the Bohmian stream lines spin in the counterclockwise direction, $S$ must be positive. It is readily obtained through Eq. (39) by numerically integrating the Bohmian velocity around a square centered in the singularity. This procedure yields $S=+3.0012$, which is consistent with the structure of the wave function in Eq. (79).

The remaining parameters are calculated as follows: adding the squares of Eqs. (46) and (47) we obtain the equation of an ellipse

$$
\left(\frac{\mathcal{X}}{\mathcal{A}}\right)^{2}+\left(\frac{\mathcal{Y}}{\mathcal{B}}\right)^{2}=1
$$

with semi-major and semi-minor axes given by $\mathcal{A}=$ $\sqrt{|S \beta / \gamma|}, \mathcal{B}=\sqrt{|S \gamma / \beta|}$ respectively. The normalized coordinates $\mathcal{X}=X / R=\left(x-x_{j}\right) / R$ and $\mathcal{Y}=Y / R=$ $\left(y-y_{j}\right) / R$ are in a reference frame oriented along the singularity's symmetry axes and centered in the singularity. Bohmian velocities enter the calculation through 

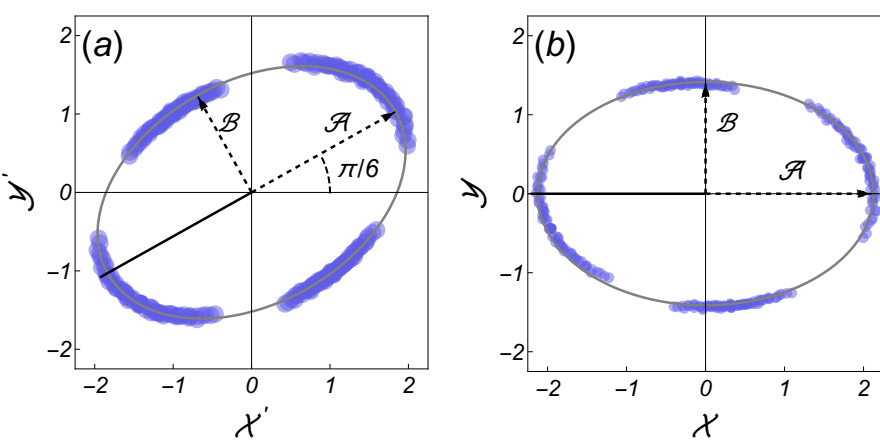

FIG. 16. a) Normalized coordinates $\mathcal{X}^{\prime}, \mathcal{Y}^{\prime}$ in the standard frame and b) normalized coordinates $\mathcal{X}, \mathcal{Y}$ in the frame aligned with the singularity's symmetry axes. The symmetry axes form an angle of $\nu=32.31^{\circ}=0.5640 \mathrm{rad} \approx \pi / 6 \mathrm{rad}$ with the $\mathcal{X}^{\prime}$-axis. In both frames the fit is indicated with a solid line.

the normalising length, given by

$$
R=\sqrt{\frac{\hbar}{m}} \sqrt[4]{\left(\frac{x-x_{j}}{v_{y}^{K}}\right)^{2}+\left(\frac{y-y_{j}}{v_{x}^{K}}\right)^{2}}
$$

As the orientation of the singularity is yet unknown we first calculate $\left(\mathcal{X}^{\prime}=X^{\prime} / R\right.$ and $\mathcal{Y}^{\prime}=Y^{\prime} / R$ in the standard frame. It is worthwhile to notice that the variables $R$ and consequently $\mathcal{X}^{\prime}$ and $\mathcal{Y}^{\prime}$ can be fully computed from the Bohmian velocity map. Fig. 16 (a) shows a plot of the points $\left(\mathcal{X}^{\prime}, \mathcal{Y}^{\prime}\right)$ in the standard frame. From the tilt of the ellipse we calculate the angle formed by the singularity's symmetry axes and the standard frame. The angle that best aligns the ellipse is $\nu=32.31^{\circ}=0.5640 \mathrm{rad} \approx \pi / 6 \mathrm{rad}$. After a $32.31^{\circ}$ rotation, the variables in the singularity frame take the form $\mathcal{X}=\mathcal{X}^{\prime} \cos \nu+\mathcal{Y}^{\prime} \sin \nu$ and $\mathcal{Y}=\mathcal{Y}^{\prime} \cos \nu-\mathcal{X}^{\prime} \sin \nu$. The new variables are plotted in Fig. 16 (b).

By fitting Eq. (91) (see Fig. 17) we find the semi-major and semi-minor axes $\mathcal{A}=2.105$ and $\mathcal{B}=1.418$. The fitted curve is shown in Figs. 16 (a) and (b) with a solid line. The topological charge $S$ can be newly calculated as $S=\mathcal{A B}=2.985$. Finally, the ratio of the $\gamma$ to the $\beta$ parameters can be determined from $|\beta / \gamma|=\mathcal{A} / \mathcal{B}=$ 1.485 .

All of these results are consistent with the structure of the wave function (79).

\section{CONCLUSIONS}

We have investigated the relation between phase singularities and the internal rotational dynamics in electron beams subject to a constant and uniform magnetic field. To understand different singularity configurations we have studied superpositions of LG modes having different angular momenta. In particular we have used HG beams and their positive and negative angular momentum parts. BEC fields where also examined given their

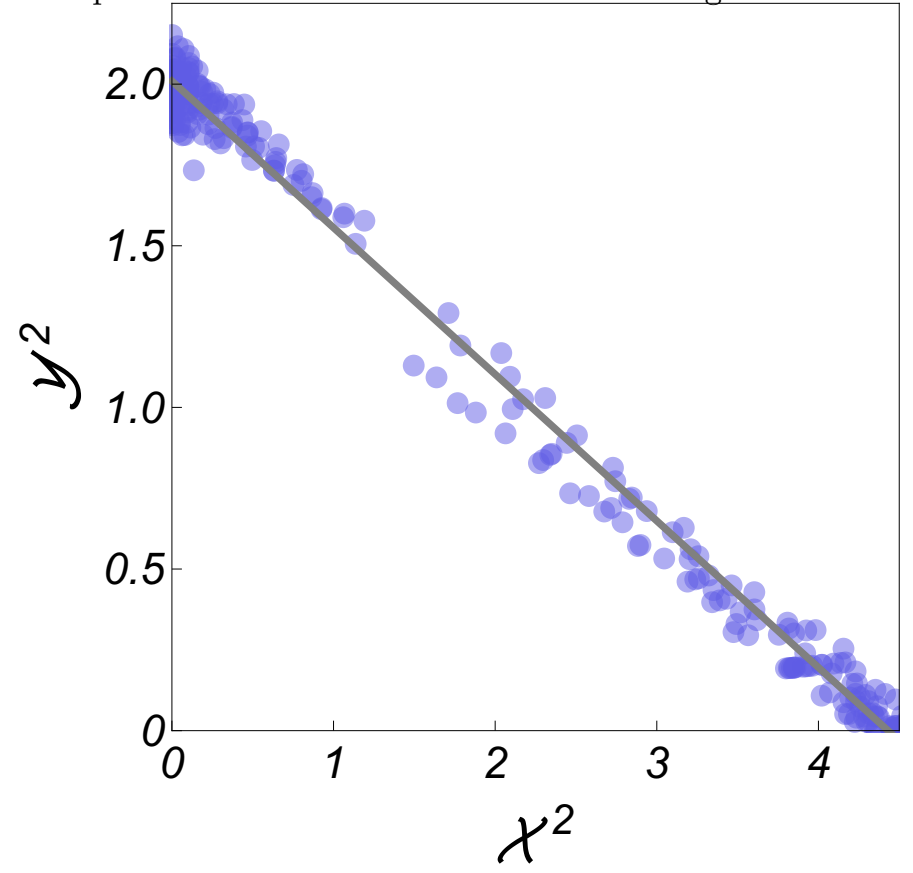

FIG. 17. $\left(X^{\prime}\right)^{2}$ vs. $\left(Y^{\prime}\right)^{2}$ and fit $\left(Y^{\prime}\right)^{2}=-m\left(X^{\prime}\right)^{2}+c$, $m=-0.441762, c=1.99527$

regular singularity structures. We have demonstrated that the wave function's mathematical form in the vicinity of a singularity plays a key role in shaping the velocity profile of the Bohmian streamlines. The topological charge, the main parameter defining the singularity's mathematical structure, can be analytically calculated from the lowest non-vanishing order of the wave function expanded around the singularity's position. Conversely, we show that the shape of the Bohmian stream lines can be used to estimate the mathematical structure of the singularity. The method developed here could lead to an experimental procedure to obtain the relevant parameters of the electron's wave function starting from a map of the Bohmian velocities.

\section{ACKNOWLEDGMENTS}

We gratefully appreciate the financial support of "Departamento de Ciencias Básicas UAM-A" grant numbers 2232214 and 2232215. J. C. Sandoval-Santana and V. G. Ibarra-Sierra would like to acknowledge the support received from the "Becas de Posgrado UAM" scholarship numbers 2151800745 and 2112800069. 
[1] V. Fock, Zeitschrift für Physik 47, 446 (1928).

[2] C. G. Darwin, Mathematical Proceedings of the Cambridge Philosophical Society 27, 86 (1931).

[3] L. Landau and E. Lifshitz, "Quantum mechanics, nonrelativistic theory," (Pergamon, Oxford, 1977) Chap. XV, pp. 456-459, 3rd ed.

[4] K. Klitzing, G. Dorda, and M. Pepper, Phys. Rev. Lett. 45, 494 (1980).

[5] T. Holstein, Phys. Rev. B 8, 2649 (1973).

[6] F. F. Fang and J. Stiles, Phys. Rev. 174, 174 (1968).

[7] R. B. Laughlin, Phys. Rev. Lett. 50, 1395 (1983).

[8] K. Y. Bliokh, Y. P. Bliokh, S. Savel'ev, and F. Nori, Phys. Rev. Lett. 99, 190404 (2007).

[9] B. J. McMorran, A. Agrawal, I. M. Anderson, A. A. Herzing, H. J. Lezec, J. J. McClelland, and J. Unguris, Science 331, 192 (2011), http://science.sciencemag.org/content/331/6014/192.full.p

[10] M. Uchida and A. Tonomura, NATURE 464, 737 (2010).

[11] P. Schattschneider, T. Schachinger, M. Stoeger-Pollach, S. Loeffler, A. Steiger-Thirsfeld, K. Y. Bliokh, and F. Nori, NATURE COMMUNICATIONS 5 (2014), $10.1038 /$ ncomms 5586 .

[12] V. Grillo, G. Carlo Gazzadi, E. Karimi, E. Mafakheri, R. W. Boyd, and S. Frabboni, Applied Physics Letters 104, 043109 (2014), http://dx.doi.org/10.1063/1.4863564.

[13] J. Verbeeck, H. Tian, and P. Schattschneider, NATURE 467, 301 (2010).

[14] V. Grillo, G. C. Gazzadi, E. Mafakheri, S. Frabboni, E. Karimi, and R. W. Boyd, Phys. Rev. Lett. 114, 034801 (2015).

[15] V. Grillo, E. Karimi, G. C. Gazzadi, S. Frabboni, M. R. Dennis, and R. W. Boyd, Phys. Rev. X 4, 011013 (2014).

[16] N. Voloch-Bloch, Y. Lereah, Y. Lilach, A. Gover, and A. Arie, NATURE 494, 331 (2013).

[17] P. Schattschneider, M. Stöger-Pollach, and J. Verbeeck, Phys. Rev. Lett. 109, 084801 (2012).

[18] P. A. M. Dirac, Proceedings of the Royal Society of London A: Mathematical, Physical and Engineering Sciences 133, 60 (1931), http://rspa.royalsocietypublishing.org/content/133/821/60.full.pdf.

[19] Y. Aharonov and D. Bohm, Phys. Rev. 115, 485 (1959).

[20] J. F. Nye and M. V. Berry, Proceedings of the Royal Society of London A: Mathematical, Physical and Engineering Sciences 336, 165 (1974), http://rspa.royalsocietypublishing.org/content/336/1605/165.full.pd

[21] M. V. Berry, Proceedings of the Royal Society of London A: Mathematical, Physical and Engineering Sciences 392, 45 (1984), http://rspa.royalsocietypublishing.org/content/392/1802/45.full.pdf.

[22] T. Schachinger, S. Lffler, M. Stger-Pollach, and P. Schattschneider, Ultramicroscopy 158, 17 (2015).

[23] G. Gbur and T. D. Visser, Optics Communications 222 , 117 (2003).

[24] W. Löffler, D. J. Broer, and J. P. Woerdman, Phys. Rev. A 83, 065801 (2011).

df[25] S. Lloyd, M. Babiker, and J. Yuan, Phys. Rev. Lett. 108, 074802 (2012).

[26] C. R. Greenshields, S. Franke-Arnold, and R. L. Stamps, New Journal of Physics 17, 093015 (2015).

[27] J. Yuan, S. M. Lloyd, and M. Babiker, Phys. Rev. A 88, 031801 (2013).

[28] C. R. Greenshields, R. L. Stamps, S. Franke-Arnold, and S. M. Barnett, Phys. Rev. Lett. 113, 240404 (2014).

[29] A. Wnsche, Journal of Computational and Applied Mathematics 133, 665 (2001), 5th Int. Symp. on Orthogonal Polynomials, Special Functions and their Applications.

[30] K. Y. Bliokh, P. Schattschneider, J. Verbeeck, and F. Nori, Phys. Rev. X 2, 041011 (2012).

[31] G. Molina-Terriza, J. P. Torres, and L. Torner, Phys. Rev. Lett. 88, 013601 (2001).

[32] K. W. Madison, F. Chevy, W. Wohlleben, and J. Dalibard, Phys. Rev. Lett. 84, 806 (2000).

[33] S. Kocsis, B. Braverman, S. Ravets, M. J. Stevens, R. P. Mirin, L. K. Shalm, and A. M. Steinberg, Science 332, 1170 (2011), http://science.sciencemag.org/content/332/6034/1170.full.pdf.

[34] K. Y. Bliokh, A. Y. Bekshaev, A. G. Kofman, and F. Nori, New Journal of Physics 15, 073022 (2013). 